\title{
PERFORMANCE SCHWEIZERISCHER VERWALTUNGSRÄTE ANHAND DER AKTIENKURSENTWICKLUNG
}

\author{
erscheint in Finanzmarkt und Portfolio Management
}

\section{$1 \quad$ Einleitung}

In den letzten Jahren haben zahlreiche Führungskrisen die Unternehmenslandschaft weltweit und in der Schweiz erschüttert und damit Fragen der Corporate Governance zu einem hochaktuellen Diskussionsthema sowohl in Politik als auch in der breiten Öffentlichkeit werden lassen. Die Leistungen von Führungskräften werden plötzlich hinterfragt, wobei Kritik oftmals an Einzelpersonen in der obersten Unternehmensführung laut wird. Dabei stellt sich die Frage nach der Messung der Performance von Führungskräften. Dieser Beitrag befasst sich deshalb mit der Messung der Performance von Verwaltungsräten in der Schweiz.

In der bisherigen Literatur ist die Leistung der einzelnen Mitglieder von Verwaltungsräten - anders als beispielsweise Portfoliomanager - kaum quantitativ betrachtet worden. Die Qualität einzelner Mitglieder wird meist nur auf Grund von Umfragen ermittelt, wie z.B. in der Studie von Knight Gianella (2002) für die Schweiz. Ein Grund für die fehlende Beachtung ist sicherlich die Tatsache, dass der effektive Beitrag einzelner Mitglieder zu den Entscheiden und Beschlüssen des Verwaltungsrates für Aussenstehende nicht einzusehen und schwer messbar ist. Gemäss der rechtlichen Haftung nach Art. 754 Abs. 1 OR, welche jedes Mitglied einzeln für absichtliche oder fahrlässige Pflichtverletzung des Gesamtverwaltungsrates verantwortlich macht, ist allerdings jedes Verwaltungsratsmitglied auch für die gesamte Unternehmensperformance verantwortlich.

Dieser Beitrag versucht, die individuelle Leistung von Verwaltungsräten zu quantifizieren und die dabei entstehenden Probleme aufzuzeigen, indem einerseits nicht das Verwaltungsratsgremium als ganzes, sondern das einzelne Mitglied im Zentrum der Untersuchung steht und andererseits eine unternehmensübergreifende Betrachtungsweise gewählt wird, bei der die Performance einer Person anhand der Performance aller Verwaltungsratsmandate dieser Person beurteilt wird. Folgende Fragestellungen sollen untersucht werden:

- Wer hat in der Schweiz mit Verwaltungsratsmandaten bei verschiedenen Gesellschaften und über einen längeren Zeitraum eine überdurchschnittliche Performance erzielt?

- Ist die Performance von Verwaltungsratsmitgliedern nachhaltig? Gibt es also Verwaltungsräte, welche systematisch über mehrere Untersuchungsperioden eine überdurchschnittliche Leistung ausweisen können? Könnte also aus der Zusammensetzung von Verwaltungsräten mit

\footnotetext{
${ }^{1}$ Schweizerisches Institut für Banken und Finanzen, Universität St. Gallen, Rosenbergstrasse 52, 9000 St. Gallen, Tel. +41 71 224-7090. Email: manuel.ammann@unisg.ch, heinrich.vonwyss@unisg.ch. Wir danken Beat Bernet, Ralf Seiz, Michael Verhofen und Heinz Zimmermann sowie zwei anonymen Gutachtern für hilfreiche Kommentare.
} 
bestimmten Personen gar auf die Performance der Aktie einer Unternehmung geschlossen werden?

- Schneiden besonders aktive, respektive begehrte Verwaltungsräte in Bezug auf die Performance besser ab als andere?

Um diese Fragen beantworten zu können, muss die Leistung eines Verwaltungsrates objektiv bestimmt werden können. Dafür kommen eine Reihe von Kennzahlen in Frage, wie Unternehmensgewinn, Free Cashflow, Return on Investment, Economic Value Added (EVA), etc. Allen diesen Kennzahlen haftet aber der Nachteil an, dass sie erstens bis zu einem gewissen Grad von der Unternehmung selbst bestimmt werden können und zweitens sich nicht unmittelbar als Gewinn oder Verlust im Portfolio des Auftraggebers des Verwaltungsrates, also des Aktionärs, niederschlagen. So ist beispielsweise ein hoher Unternehmensgewinn für den Aktionär kaum mit Nutzen verbunden, wenn die Performance der Aktie trotzdem unterdurchschnittlich ist, weil z.B. der Markt den Unternehmensgewinn als nicht nachhaltig beurteilt. Als Massstab für die Beurteilung der Performance des Verwaltungsrates - im Sinne einer besten Approximation der tatsächlichen Performance - wird deshalb die Performance der Aktie gewählt.

Im folgenden Abschnitt wird die bisherige Forschung zusammengefasst, während in Abschnitt 3 die verwendeten Daten beschrieben werden. Abschnitt 4 befasst sich mit der Analyse der personenbezogenen Performance und der Persistenz, hierauf folgt im Abschnitt 5 die personenübergreifende Performanceanalyse. Abschnitt 6 fasst die Resultate zusammen

\section{Literaturüberblick}

Bisher wurde im Rahmen zahlreicher Untersuchungen der Zusammenhang zwischen der Charakteristik des Verwaltungsratsgremiums und der Performance der Unternehmung analysiert. Dabei stehen vor allem die Struktur, die Arbeit und die Zusammensetzung des Verwaltungsrates im Zentrum des Interesses. JOHNSON et al. (1996) unterscheiden zwischen der Führungs- und Überwachungsfunktion, der Beratungsfunktion und der Ressourcenzugangsfunktion.

Der Verwaltungsrat übt im Unternehmen eine Führungs- und Überwachungsfunktion aus. Die Ausgestaltung und Umsetzung einer effizienten Corporate Governance gehört dabei zu seinen Kernaufgaben, um das Principal-Agent-Problem zwischen Aktionären und Management zu entschärfen. Mehrere Autoren untersuchen den Zusammenhang zwischen der Grösse des Verwaltungsrates und der Unternehmensperformance: GRÜNBICHLER und OERTMANN (1995) kommen empirisch zum Ergebnis, dass mittlere Verwaltungsräte in der Schweiz tendenziell eine bessere Performance erzielen als grössere. Sie bestätigen damit das theoretische Konzept von JENSEN (1993), der von einer negativen Korrelation zwischen Unternehmenswert und Verwaltungsratsgrösse ausgeht. Er begründet dies damit, dass überdimensionierte Gremien oft vom CEO dominiert werden und dadurch keine effiziente Aufsichtsfunktion mehr wahrnehmen können. $\mathrm{Zu}$ ähnlichen Resultaten kommen Studien von YERMACK (1996) für die USA und EISENBERG et al. (1998) für Finnland.

Die Auswirkung von Doppelmandaten (Geschäftsleitungsvorsitzende, welche gleichzeitig das Amt des Verwaltungsratspräsidenten ausüben) auf die Performance sind ebenfalls ein wichtiger Forschungsgegenstand der Corporate Governance. Empirische Arbeiten kommen dabei zu unterschiedlichen Ergebnissen: RECHNER und DALTON (1991) zeigen, dass Unternehmen mit getrennten Führungsstrukturen langfristig eine bessere Performance erzielen. Nach BRICKLEY et 
al. (1997) ist eine Trennung mit erhöhten (Transaktions-)Kosten verbunden. PALMON und WALD (2002) finden je nach Unternehmensgrösse unterschiedliche Auswirkungen. Auch DAHYA und TRAVLOS (2000) plädieren für eine differenzierte Betrachtungsweise, abhängig von Firmengrösse und Beobachtungszeitpunkt. Noch weiter gehen BALIGA et al. (1996), die anhand ihrer Analyse darlegen, dass die oberste Führungsstruktur kaum Einfluss auf die langfristige Performance hat.

Die Zusammensetzung des Verwaltungsratsgremiums (im Besonderen dem Verhältnis zwischen internen und externen Personen) ist ein weiterer Untersuchungsgegenstand: Agency-Vertreter wie DONALDSON (1990) sind der Auffassung, dass gleichzeitig operativ und strategisch tätige Personen eher opportunistisch agieren und sich nicht im Sinne der Aktionäre verhalten. Sie gehen auch davon aus, dass diese Verwaltungsratsmitglieder die Leistung der Geschäftsleitung aus Loyalitätsgründen nicht objektiv beurteilen können. Aus diesen Gründen bevorzugen sie Personen von aussen, die über eine kritische Distanz zum Unternehmen verfügen. Andererseits weist SCHAFFER (2002) im Gegensatz dazu auf Probleme hin, welche mit Outsider-dominierten Verwaltungsratsgremien verbunden sind: Die Mandatsträger haben nicht genügend Zeit und zuwenig Informationen um ihre Aufgaben wahrzunehmen. Auch hier kommen empirische Untersuchungen zu uneinheitlichen Ergebnissen: HERMALIN und WEISBACH (1991) finden keinen Zusammenhang zwischen der Unternehmensperformance und dem Anteil externer Verwaltungsratsmitglieder. BAYSINGER und BUTLER (1985) folgern jedoch, dass Unternehmen bessere Resultate erzielen, wenn mehrheitlich externe Personen im Aufsichtsgremium vertreten sind. Diese Feststellung stützen ROSENSTEIN und WYATT (1990) mit ihrem Befund, wonach Investoren positiv auf die Wahl von unabhängigen Personen reagieren.

Die Beratungsfunktion bezieht sich auf die Unterstützung der Geschäftsleitung durch den Verwaltungsrat in strategischen Angelegenheiten. Nach MACE (1971) und LORSCH und MACIVER (1989) betrachten viele Verwaltungsratsmitglieder dies als die Schlüsselfunktion ihrer Tätigkeit. Der Verwaltungsrat muss die Strategie nicht selber entwickeln, muss jedoch besorgt sein, dass erfolgsversprechende Strategien vorhanden sind, wie WEIBEL (1998) festhält. Um den Einfluss auf die Performance beurteilen zu können, stellt sich in diesem Zusammenhang die Frage, in welchem Ausmass die Verwaltungsratsmitglieder in den strategischen Entscheidungsprozess einbezogen werden. Da die Beratungsfunktion von zahlreichen Faktoren abhängt und unternehmens- und verwaltungsratsspezifisch sehr unterschiedlich wahrgenommen wird, können allerdings kaum allgemein gültige Aussagen gemacht werden. JUDGE und ZEITHAML (1992) kommen zum Schluss, dass der Einbezug des Verwaltungsrates von der Anzahl interner Personen im Verwaltungsrat, der Grösse des Gremiums, der Diversifikation und dem Alter des Unternehmens abhängt. Sie zeigen, dass sich eine stärkere Involvierung des Verwaltungsrates positiv auf die Performance auswirkt.

Besondere Aufmerksamkeit erhalten in dieser Rolle die externen Verwaltungsratsmitglieder und deren Nutzen als Berater der Geschäftsleitung. Dieser Gesichtspunkt ist auch für diese Arbeit interessant, da Verwaltungsräte mit mehreren Mandaten selten in mehr als einer Firma gleichzeitig operativ tätig sind. Anhand einer Untersuchung von High-Tech-Unternehmen können ROSENSTEIN et al. (1993) aufzeigen, dass externe Verwaltungsratsmitglieder in der Anfangsphase eines Unternehmens aufgrund ihres Wissens und ihrer Erfahrung einen wertsteigernden Einfluss haben. Dieser Wert nimmt allerdings mit der Zeit kontinuierlich ab. 
Die auf PFEFFER und SALANCIK (1978) zurückgehende Ressourcenzugangsfunktion sieht die Verwaltungsratsmitglieder als Hilfsmittel zum erleichterten Zugang zu kritischen Ressourcen. Personelle Verflechtungen zwischen rechtlich selbständigen Unternehmen über Verwaltungsräte und Netzwerke sind ein direkter Ausdruck dieser Sichtweise. Dabei geht es in erster Linie um Kollusion (das Zusammenwirken von Unternehmen, um den Wettbewerb zu beschränken), Reduktion der Umweltunsicherheit und um die Legitimierungsfunktion von Verflechtungen, wie PFANNSCHMIDT (1993) und MIZRUCHI (1996) festhalten. Nach PENNINGS (1980) und BURT (1983) beeinflusst Kollusion via Verwaltungsratsgremien die Profitabilität der betroffenen Firmen kaum. Zu einem anderen Ergebnis kommt CARRINGTON (1981), der eine positive Relation zwischen Performance und Verflechtung von Wettbewerbern via Verwaltungsrat findet. Bei der Reduktion der Umweltunsicherheit geht es um eine Absicherung gegenüber anderen Anspruchsgruppen. Im Gegensatz zur Kollusion nehmen hier also nicht Wettbewerber, sondern Kunden, Lieferanten und insbesondere Eigen- und Fremdkapitalvertreter Einsitz im Verwaltungsratsgremium. Die Idee dabei ist, dass nicht kontrollierbare Faktoren, die das eigene Verhalten beeinflussen, mit in die Entscheidungsstruktur einbezogen werden und somit potenzielle Unsicherheitsaspekte reduziert werden können. Auch hierzu gibt es in der Literatur keinen Konsens über positive oder negative Performanceeffekte einer solchen Konstellation. Einerseits beobachten PENNINGS (1980), CARRINGTON (1981) und BURT (1983) einen leicht positiven, aber schwachen Zusammenhang zwischen Profitabilität und der Vertretung von Anspruchsgruppen im Gremium, andererseits kommen FLIGSTEIN und BRANTLEY (1992) genau zum umgekehrten Schluss.

Bei der Legitimierungsfunktion geht es nicht in erster Linie um Vorteile einer Allianz mit anderen Unternehmen, sondern um das Prestige, welches mit gewissen Personen, respektive deren Verwaltungsratsmandaten verbunden ist. So signalisieren nach MIZRUCHI (1996) und PFANNSCHMIDT (1993) Personen, die bereits mehrere Mandate haben, das Vertrauen von verschiedenen unterschiedlichen Gremien. Dies impliziert hohe Kompetenz plus Zugang zu einem Netzwerk von Kontakten und sollte sich indirekt auch positiv auf die Performance auswirken. Da sich eine Abgrenzung zum vorhergehenden Ansatz in der Praxis schwierig gestaltet und die Vorhersagen eng damit verknüpft sind, fehlen Untersuchungen, die den Performanceeffekt isoliert auf diesen Fall betrachten.

Abgesehen von den drei genannten Erklärungsansätzen gibt es in der Literatur weitere Begründungen für Verflechtung von Verwaltungsratsgremien:

(1) Sozialer Zusammenhalt: Verflechtungen repräsentieren gemäss MIZRUCHI (1996) den sozialen Zusammenhalt in der Oberklasse, d.h. neue Verwaltungsratsmitglieder werden aufgrund ihrer Zugehörigkeit zu einer besonderen Bevölkerungsschicht gewählt.

(2) Karriere und Macht: Hier verschiebt sich die Perspektive zu denjenigen Personen, die sich in Verwaltungsräte wählen lassen. Motivation zur Übernahme mehrerer Mandate sind nach ZAJAC (1988) ökonomische Anreize, Prestige und neue Kontakte.

(3) Geringe Konzentration von Führungskräften: Hierbei handelt es sich um eine typisch schweizerische Begebenheit, denn gemessen an der Einwohnerzahl sind relativ viele Unternehmen von internationaler Bedeutung in der Schweiz kotiert. Die kleine Elite in Wirtschaft und Politik erfordert deshalb oft Mehrfachfunktionen, nicht nur in Unternehmen, sondern auch in politischen, 
militärischen und kulturellen Gremien. Wie SCHWARZ (2001) festhält, ist ein dichtes Netzwerk, oft auch als „Filz“ bezeichnet, die logische Konsequenz davon.

In der Praxis gestaltet sich eine klare Abgrenzung von Motiven für Verflechtungen schwierig, weshalb verschiedene Autoren die Erfolgswirksamkeit von Verflechtungen nur generell untersuchen: DOWEN (1995) untersucht in amerikanischen Grossunternehmen den Zusammenhang zwischen der durchschnittlichen Anzahl Mandate pro Verwaltungsratsmitglied und der Unternehmensperformance. Er kommt zum Ergebnis, dass Firmen, deren Verwaltungsräte eine hohe durchschnittliche Anzahl Mandate bei anderen Unternehmen aufweisen, eine bessere Performance erzielen als Firmen mit geringen Verflechtungen. Zum umgekehrten Schluss kommt PFANNSCHMIDT (1993) für Deutschland: Er findet keinen statistisch signifikanten Zusammenhang zwischen Performance und dem Ausmass der Verflechtung.

\section{Datengrundlage}

Die etwa 200'000 Verwaltungsräte von Schweizer Firmen wurden auf 95 Personen eingeschränkt, indem nur Personen berücksichtigt wurden, die im Beobachungszeitraum vom 1. Januar 1992 bis zum 31. Dezember 2001 in mindestens zwei Firmen des Swiss Market Index (SMI) als Verwaltungsrat aktiv waren. Weil die Performance im Sinne der Abgrenzung dieser Arbeit nur bei börsengehandelten Aktiengesellschaften objektiv gemessen werden kann, fallen Personen mit Mandaten bei ausschliesslich nicht-kotierten Gesellschaften weg. Von den übrigen Personen sind vor allem solche von Interesse, die bei verschiedenen bedeutenden Gesellschaften im Verwaltungsrat vertreten sind oder waren, da dies einerseits ein Indiz für Einfluss und Kompetenz darstellt und andererseits die Performance dadurch nicht nur von der Tätigkeit in einer einzigen Gesellschaft abhängt.

Am Ende des Beobachtungszeitraumes umfasste der SMI 26 Gesellschaften. Da der SMI vierteljährlich (oder in Ausnahmefällen auch laufend) angepasst wird, gibt es regelmässig Veränderungen in der Indexzusammensetzung. Massgebend ist die Mandatstätigkeit bei einer Gesellschaft, während der Zeit, in der sie dem SMI angehört.

Das Erhebungsmerkmal „mindestens zwei Firmen“ bezieht sich nicht zwingend auf verschiedene Mandate im gleichen Jahr. Es reicht aus, bei zwei verschiedenen Gesellschaften zu unterschiedlichen Zeitpunkten Mitglied des Verwaltungsrates gewesen zu sein. Mandate bei Gesellschaften vor und nach einer Fusion werden nicht doppelt gewertet.

Für alle Personen der Stichprobe werden von 1992 bis 2001 auf jährlicher Basis Daten erhoben. Massgebend sind dabei die Angaben aus dem Verzeichnis der Verwaltungsräte (1991-2000). Da das Verzeichnis nur einmal pro Jahr publiziert wird, stellt sich die Frage, wie und wann Veränderungen in der Zusammensetzung von Verwaltungsräten berücksichtigt werden sollen. Zwar schliessen die meisten Unternehmen das Geschäftsjahr per 31. Dezember ab, Mutationen im Verwaltungsrat finden aber in der Regel erst an der ordentlichen Generalversammlung statt. Da diese Generalversammlungen erst nach Veröffentlichung des Jahresabschlusses durchgeführt werden können, ergibt sich eine Abweichung zwischen Verantwortung und Mandatstätigkeit. In diesem Zusammenhang ist aber auch fraglich, inwiefern ein neu gewähltes Verwaltungsratsmitglied noch einen Einfluss auf das laufende Geschäftsjahr haben kann. Planung und Budgetierung werden bereits im Vorjahr festgelegt und zudem ist ein Viertel bis ein Drittel des laufenden Jahres bereits vorbei. Dazu kommt normalerweise eine gewisse Einarbeitungszeit. Aus diesen Grün- 
den und wegen mangelnder Verfügbarkeit von detaillierten Daten in Bezug auf Beginn und Ende der Verwaltungsratstätigkeit wird folgende Regelung angewandt: Die im Verzeichnis der Verwaltungsräte aufgelisteten Mandate werden jeweils für den Zeitraum vom 1. Januar bis 31. Dezember des darauf folgenden Jahres als performancerelevant betrachtet. Das heisst einerseits, dass neue Verwaltungsratsmitglieder erst im Folgejahr die Unternehmensperformance mittragen und andererseits, dass zurückgetretene Verwaltungsräte das Jahresergebnis im Jahr ihres Rücktritts mitverantworten. Dies bringt folgende Vorteile mit sich:

- Auswirkungen von strategischen Entscheiden des Verwaltungsrates werden zuverlässiger erfasst, da deren Umsetzung normalerweise mehrere Wochen oder Monate dauert.

- Die Diskrepanz zwischen Amtsantritt und Geschäftsjahr fällt nicht ins Gewicht, da erst das Folgejahr betrachtet wird.

Diese Handhabung ist auch für Gesellschaften zweckmässig, deren Geschäftsjahr nicht per 31 . Dezember endet: Aufgrund der Verzögerung zwischen den Entscheiden des Verwaltungsrates und deren Auswirkungen auf die langfristige Performance, ist ein gewisser Lag für die Performanceberechnung durchaus plausibel. Es könnte jedoch argumentiert werden, dass der Einfluss eines neuen Verwaltungsrates auf die Aktienkursentwicklung noch später einsetzt und dafür nach dem Aussscheiden länger anhält. Auf der anderen Seite ist auch denkbar, dass die Ankündigung einer neuen Einsitznahme im Verwaltungsrat bereits einen Announcementeffekt im Aktienkurs bewirkt und somit während der eigentlichen Verweildauer des Verwaltungsrates in der Unternehmung nurmehr durchschnittliche Aktienrenditen erzielt werden.

Erfasst werden nur Mandate von Unternehmen, die an der Schweizer Börse gelistet sind oder gelistet waren. Am 31. Dezember 2001 waren dies 263 Gesellschaften. Bei Titeln, die während dem Erhebungszeitraum neu kotiert oder dekotiert wurden, beschränkt sich die Erhebung auf den Kotierungszeitraum. Das Jahr der Neu- oder Dekotierung wird von der Betrachtung ausgeschlossen, da sonst im Hinblick auf die Anzahl Mandate und die Performancemessung Verzerrungen resultieren würden. Dies weil Neu- und Dekotierungen in der Regel während des Jahres stattfinden und somit nicht ganze Mandatsjahre umfassen.

Die Amtstätigkeit der 95 ausgewählten Personen verteilt sich auf 134 verschiedene Gesellschaften. Von diesen Gesellschaften waren am 31. Dezember 2001 insgesamt 107 gelistet; deren Börsenkapitalisierung entspricht über 95\% der Marktkapitalisierung des Schweizer Aktienmarktes. Interessanterweise gehören mit Ausnahme von Kudelski sämtliche SMI Gesellschaften dazu, was aufgrund der Stichprobenauswahl bedeutet, dass in jedem dieser Unternehmen mindestens ein Verwaltungsratsmitglied ein Mandat bei einem anderen Grossunternehmen inne hat oder hatte. Dies ist ein Indikator für die ausgeprägte Verflechtung der Verwaltungsratsgremien von Schweizer Grossunternehmen.

Im Durchschnitt waren die Mandatsträger im Beobachtungszeitraum in 4.3 verschiedenen Unternehmen als Verwaltungsrat tätig. Die Häufigkeitsverteilung der Stichprobe ist in Abbildung 1 dargestellt. 
Abbildung 1: Anzahl Mandate bei verschiedenen Gesellschaften

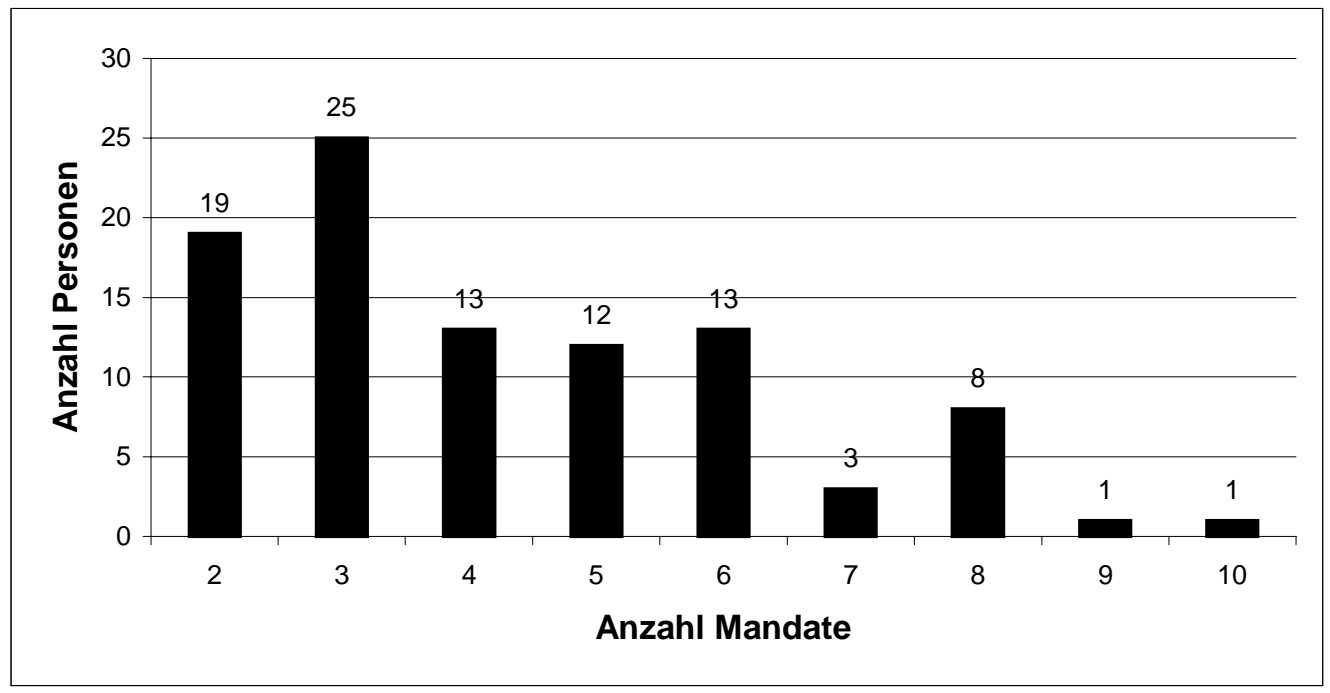

Die Stichprobe umfasst insgesamt 1984 Mandatsjahre, wobei unter Mandatsjahr eine einjährige Tätigkeit in einem Verwaltungsratsgremium verstanden wird. Die durchschnittliche Amtszeit im Untersuchungszeitraum beträgt je Mandat 4.8 Jahre. Diese Zahl darf jedoch nicht mit der generellen durchschnittlichen Amtszeit von Verwaltungsräten gleichgesetzt werden, da nicht erfasst wurde, wie lange ein Verwaltungsratsmitglied schon vor $1992 \mathrm{im} \mathrm{Amt} \mathrm{war,} \mathrm{respektive} \mathrm{wie} \mathrm{lange}$ es nach 2001 noch im Amt sein wird.

Im Durchschnitt war jeder Verwaltungsrat jährlich in 2.1 Unternehmen gleichzeitig engagiert, wobei die Beobachtungen von keinem bis zu acht gleichzeitig ausgeführten Mandaten reichen, wie Abbildung 2 zeigt. Durchschnittlich waren pro Jahr 18\% der Personen in keinem Verwaltungsrat aktiv, $23.5 \%$ in genau einem und die restlichen $58.5 \%$ in zwei oder mehr Gremien vertreten. Detaillierte Angaben zur individuellen Verwaltungsratstätigkeit der ausgewählten Personen finden sich im Anhang.

Abbildung 2: Durchschnittliche Mandatstätigkeit pro Jahr

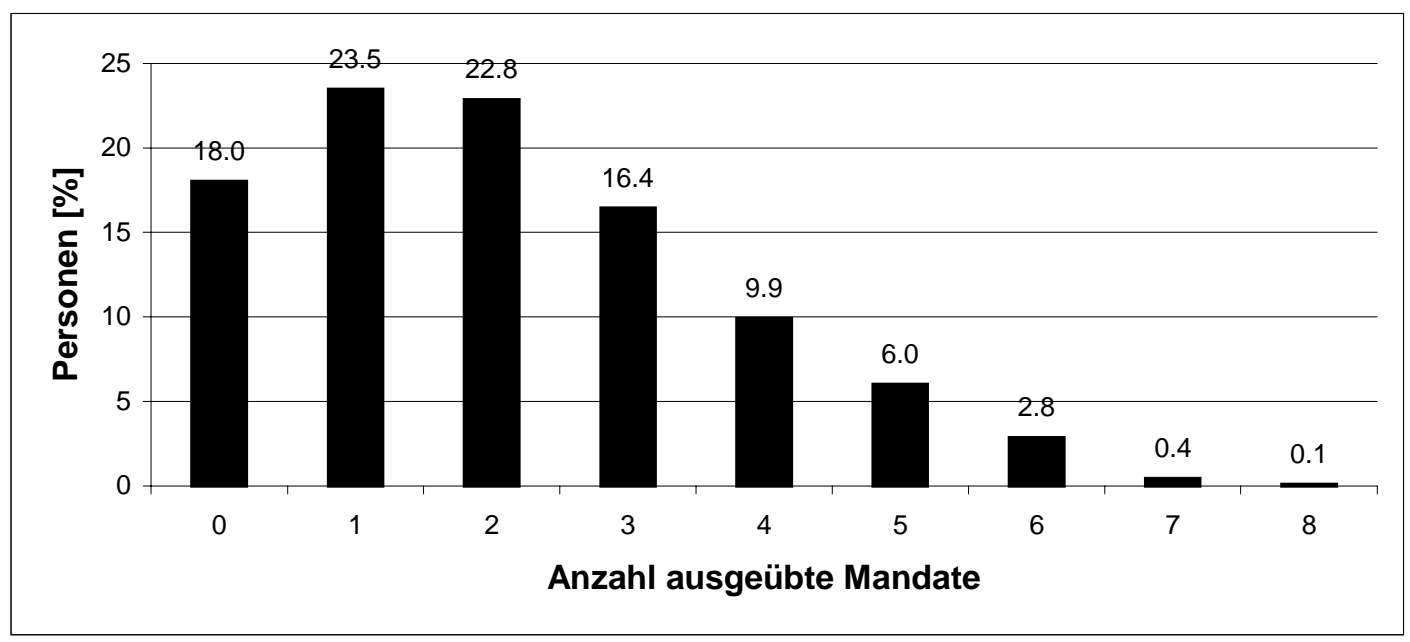


Grundlage der Performancemessung dieser Untersuchung ist die Rendite einer Gesellschaft am Kapitalmarkt. Erfasst wird der „Total Return Index“ von Datastream. Der Total Return Index ist dadurch charakterisiert, dass sämtliche Zahlungen an die Aktionäre rechnerisch reinvestiert werden. Bei Gesellschaften mit mehreren kotierten Aktienkategorien wird der liquideste Titel für die Performancemessung verwendet. Als risikoloser Zinssatz dient der 1-Monats-Euromarktzins für Schweizer Franken.

\section{Personenbezogene Performanceanalyse}

Grundsätzlich findet man in der Corporate Governance Literatur ein grosses Spektrum von verschiedenen Methoden zur Performancemessung, da der Begriff Performance oft unterschiedlich definiert wird. Generell gibt es keinen Konsens über die Auswahl geeigneter Masse für die Beurteilung der Unternehmensperformance, wie JOHNSON et al. (1996), S. 430 feststellen. Da der Verwaltungsrat in erster Linie gegenüber den Aktionären Verantwortung trägt, soll die Perspektive des Eigentümers massgebend sein. Somit bildet die Aktienrendite einer Gesellschaft die Grundlage der Performancemessung. Für die individuellen Renditen der ausgewählten Personen ist die Unternehmensrendite während der jeweiligen Verwaltungsratstätigkeit massgebend. Diese setzt sich aus Kurssteigerungen, Dividenden und sonstigen Zahlungen an die Aktionäre (beispielsweise Nennwertrückzahlungen) zusammen.

Auf eine Unterscheidung der verschiedenen Verwaltungsratsmitglieder anhand der Position (beispielsweise Verwaltungsratspräsident oder Mitglied eines Ausschusses) wird verzichtet, da die Performance analog der haftungsrechtlichen Handhabung von allen Mitgliedern im gleichen Mass mitgetragen werden muss. Die Performance soll auch nicht durch sonstige Kriterien wie Verweildauer im Verwaltungsrat, Beteiligung an der Firma, Alter, Bildung Geschlecht etc. erklärt werden.

\subsection{Verwaltungsratsmandate als Portfolio}

Den Berechnungen in diesem Abschnitt liegt die Überlegung zu Grunde, dass die zu betrachtenden Personen im Verlauf des Beobachtungszeitraums über ein wechselndes Portfolio von Verwaltungsratsmandaten verfügen. Als Konsequenz soll die Performance aufgrund der Abfolge der jeweiligen Zusammensetzung des individuellen Portfolios analysiert werden. Dadurch kann aufgezeigt werden, welche Personen über den gesamten Zeitraum über die effektivsten Portfolios verfügt haben und dadurch eine bessere Performance erzielt haben als andere. Mit anderen Worten wird festgestellt, welcher Verwaltungsrat für einen Aktionär die beste „Wertanlage“ gewesen wäre. Zwar haben die Mandatsträger nur in einem beschränkten Ausmass Einfluss auf die Zusammensetzung ihrer Portfolios, dafür üben sie in ihren Rollen als Verwaltungsrat umso mehr Gewicht auf die Wertschätzung einer Firma am Kapitalmarkt und somit auf die Performance aus. Die zu analysierenden Personen haben Phasen, in denen sie mehreren Gremien angehört haben, Abschnitte mit nur einer einzelnen Mandatstätigkeit und Perioden, in denen sie in keiner Gesellschaft als Verwaltungsrat amtierten. Die Renditen für diese unterschiedlichen Perioden werden im vorliegenden Abschnitt folgendermassen berechnet:

- Perioden mit nur einem Mandat: Die Rendite der betreffenden Gesellschaft ist massgebend für die Portfoliorendite während diesem Zeitraum.

- Perioden mit mehreren Mandaten gleichzeitig: Es wird das arithmetische Mittel der verschiedenen Unternehmensrenditen verwendet. Dabei stellt sich die Frage, inwiefern eine Gleich- 
gewichtung für die Berechnung angemessen ist. Als mögliche Alternative wäre eine Gewichtung nach Marktwert der jeweiligen Unternehmen vorstellbar. Allerdings würden wegen der sehr unterschiedlichen Firmengrössen im Schweizer Markt Mandate bei mittleren und kleineren Gesellschaften marginalisiert. Eine 100fache Übergewichtung gewisser Mandate würde in keinem Verhältnis mit der Verantwortung und dem Aufwand der Mandatstätigkeit stehen.

- Perioden ohne Verwaltungsratstätigkeit: Die Rendite des Benchmarks wird als erzielte Rendite eingesetzt.

Diesen Bestimmungen liegt die Überlegung zu Grunde, dass das Portfoliovermögen jeweils ana$\log$ der Verwaltungsratstätigkeit umgeschichtet und vollständig reinvestiert wird; während dem gesamten Betrachtungszeitraum gibt es somit keine Ein- und Auszahlungen aus den jeweiligen Portfolios. Für alle Verfahren wird mit stetigen Renditen gerechnet.

\section{Portfoliorendite relativ zum Gesamtmarkt}

Die erzielte Überrendite berechnet sich als Differenz zwischen der Portfoliorendite $\left(\mathrm{r}_{\mathrm{Pf}}\right)$ und der Benchmarkrendite $\left(\mathrm{r}_{\mathrm{M}}\right)$ :

$$
\text { Überrendite }=r_{P f}-r_{M}
$$

Da in dieser Untersuchung ausschliesslich an der Schweizer Börse (SWX) kotierte Unternehmen betrachtet werden, ist der Swiss Performance Index (SPI), der relevante Benchmark. Einerseits umfasst er alle an der SWX kotierten Gesellschaften,[1] andererseits wird er nach der TotalReturn-Methode mit reinvestierten Dividenden berechnet.

Für Perioden ohne Mandatstätigkeit wurde eine Überrendite von $0 \%$ angenommen. Dadurch werden Personen, welche in guten oder schlechten Börsenjahren nicht aktiv waren, weder bevorzugt noch benachteiligt.[2]

Folgende Einschränkung muss bei der Interpretation der Ergebnisse gemacht werden: Das Resultat beinhaltet Faktoren, die der Verwaltungsrat einer Unternehmung nicht beeinflussen kann. Dazu gehört insbesondere die Branchenentwicklung, die in der Regel die Unternehmenswertentwicklung zu einem hohen Grad mitbestimmt.

Auf eine Performanceanlyse relativ zur Branche wurde an dieser Stelle aus folgenden Gründen verzichtet:[3]

- Schlechte Diversifikation einiger Indizes: Beispielsweise beträgt die Gewichtung von Nestlé innerhalb der Nahrungsmittelwerte des SPI aufgrund des hohen Marktwertes über 90\%. Nestlé kann somit nur eine sehr geringe Über- oder Unterrendite relativ zur Branche erzielen.

- Mangelnde Homogenität: Die beiden Sammelbecken „verschiedene Dienstleistungen“ und „übrige Industrien“ des SPI sind keine homogenen Indizes. Es kann kaum von „Branche“ gesprochen werden.

- Die Verwendung eines internationalen Branchenindizes könnte das Problem der Gewichtung und Homogenität entschärfen, ergäbe dafür aber neue Probleme. Insbesonder würden Ländereffekte in die Performancemessung einfliessen, wie DRUMMEN und ZIMMERMANN 
(1992) feststellen. Da in der vorliegenden Stichprobe nur Schweizer Verwaltungsratsmandate untersucht werden, ist dies unerwünscht.

\section{Volatilität}

Die Volatilität für die betrachteten Mandatsportfolios wird als absolute Volatilität aufgrund der wöchentlichen stetigen Portfoliorenditen berechnet und anschliessend annualisiert.

Im Vergleich zum SPI ergeben sich folgende annualisierten Überrenditen und Volatilitäten für die 95 Verwaltungsräte im Sample:

Tabelle 1: Portfolioperformanceanalyse: Relative Rendite zum Gesamtmarkt (SPI)

\begin{tabular}{|c|c|c|c|c|c|c|c|}
\hline Rang & Name & Überrendite & Volatilität & Rang & Name & Überrendite & Volatilität \\
\hline 1 & Sarasin B. & $8.93 \%$ *** & $30.70 \%$ & 49 & Angst C. & $-2.55 \% \%^{\star * *}$ & $14.28 \%$ \\
\hline 2 & Gasser R. & $6.97 \%$ *** & $19.68 \%$ & 50 & Tschopp T. & $-2.61 \% \%^{\star \star \star}$ & $20.31 \%$ \\
\hline 3 & Schäuble R. & $6.15 \% \%^{* * *}$ & $21.26 \%$ & 51 & Doerig $\mathrm{H}$. & $-2.63 \%$ *** & $16.06 \%$ \\
\hline 4 & Junger M. & $5.43 \%$ *** & $26.24 \%$ & 52 & Gugler A. & $-2.73 \% \%^{\star \star \star}$ & $14.51 \%$ \\
\hline 5 & Muller G. & $4.44 \%$ *** & $25.10 \%$ & 53 & Lippuner H. & $-2.96 \% \%^{* * *}$ & $23.77 \%$ \\
\hline 6 & Sigg $\mathrm{H}$. & $4.24 \%^{\star \star \star}$ & $24.66 \%$ & 54 & Von Finck A. & $-3.13 \% * \star \star *$ & $19.19 \%$ \\
\hline 7 & Gut R. & $4.22 \%$ *** & $21.63 \%$ & 55 & Zumstein J. & $-3.13 \% \%^{\star \star \star}$ & $9.65 \%$ \\
\hline 8 & Languetin P. & $4.02 \%$ *** & $24.09 \%$ & 56 & Schweizer R. & $-3.21 \% \%^{* * *}$ & $25.48 \%$ \\
\hline 9 & Blocher C. & $3.64 \%$ *** & $16.11 \%$ & 57 & Gisling J. & $-3.40 \% \%^{* * *}$ & $23.37 \%$ \\
\hline 10 & Bremi U. & $3.35 \%$ *** & $24.89 \%$ & 58 & Hüppi R. & $-3.77 \%$ \%** & $26.78 \%$ \\
\hline 11 & Krauer $\mathrm{A}$. & $3.24 \%$ *** & $21.06 \%$ & 59 & Senn N. & $-4.07 \% \%^{* \star *}$ & $29.23 \%$ \\
\hline 12 & Kielholz W. & $2.39 \%$ *** & $12.56 \%$ & 60 & Müller-Berghof B. & $-4.49 \% \%^{* * *}$ & $20.49 \%$ \\
\hline 13 & Ebner M. & $2.39 \%$ *** & $21.41 \%$ & 61 & Milliet F. & $-4.83 \%$ *** & $12.44 \%$ \\
\hline 14 & Studer R. & $2.28 \%$ *** & $23.06 \%$ & 62 & Bodmer $\mathrm{H}$. & $-4.90 \%^{\star \star *}$ & $27.79 \%$ \\
\hline 15 & Albers U. & $2.24 \%$ *** & $26.41 \%$ & 63 & Ackermann J. & $-5.02 \% \%^{\star \star *}$ & $26.52 \%$ \\
\hline 16 & Schmidheiny S. & $2.21 \%$ *** & $21.96 \%$ & 64 & Ruepp R. & $-5.15 \%$ \%** & $15.53 \%$ \\
\hline 17 & Diehl W. & $2.14 \%$ *** & $12.37 \%$ & 65 & Meyer R. & $-5.99 \%$ *** & $23.39 \%$ \\
\hline 18 & Dunkel A. & $1.94 \%$ *** & $20.19 \%$ & 66 & Höhler G. & $-6.04 \%$ \%** & $16.86 \%$ \\
\hline 19 & Bodmer A. & $1.62 \% \%^{* *}$ & $15.81 \%$ & 67 & Pozzi A. & $-6.45 \%$ *** & $16.27 \%$ \\
\hline 20 & Böckli P. & $1.60 \%$ ** & $20.93 \%$ & 68 & Humer F. & $-6.50 \% \%^{\star \star \star}$ & $17.27 \%$ \\
\hline 21 & Frehner W. & $1.54 \%$ ** & $19.06 \%$ & 69 & Kündig M. & $-6.80 \%$ *** & $19.38 \%$ \\
\hline 22 & Vasella D. & $1.48 \%$ ** & $18.41 \%$ & 70 & Cappis M. & $-6.95 \% * \star \star *$ & $20.47 \%$ \\
\hline 23 & Marchionne S. & $1.42 \%$ ** & $11.71 \%$ & 71 & de Kalbermatten B. & $-7.11 \%^{\star \star \star}$ & $17.79 \%$ \\
\hline 24 & Grether E. & $1.26 \%{ }^{*}$ & $27.16 \%$ & 72 & Lehn J. & $-7.19 \% \%^{* \star *}$ & $21.17 \%$ \\
\hline 25 & Galliker F. & $1.07 \%$ & $10.09 \%$ & 73 & von der Crone $\mathrm{H}$. & $-7.45 \% \%^{\star \star \star}$ & $15.46 \%$ \\
\hline 26 & Küpfer P. & $0.74 \%$ & $18.58 \%$ & 74 & Leuenberger A. & $-7.73 \% \%^{* * *}$ & $24.89 \%$ \\
\hline 27 & Genillard R. & $0.66 \%$ & $24.22 \%$ & 75 & Domeniconi R. & $-7.76 \% \%^{\star \star \star}$ & $20.93 \%$ \\
\hline 28 & Gerber F. & $0.61 \%$ & $19.08 \%$ & 76 & van Wachem L. & $-7.97 \%$ \%** & $26.37 \%$ \\
\hline 29 & Jucker H. & $0.44 \%$ & $14.06 \%$ & 77 & Zbinden P. & $-8.03 \% \%^{\star \star *}$ & $42.05 \%$ \\
\hline 30 & Moret M. & $0.41 \%$ & $11.19 \%$ & 78 & Jeker R. & $-8.18 \% \%^{* \star *}$ & $20.26 \%$ \\
\hline 31 & Brabeck P. & $0.35 \%$ & $19.23 \%$ & 79 & Kissling W. & $-8.18 \% \%^{\star \star *}$ & $29.17 \%$ \\
\hline 32 & Landolt P. & $0.07 \%$ & $20.08 \%$ & 80 & Schmidheiny J. & $-9.87 \%^{* \star *}$ & $20.71 \%$ \\
\hline 33 & Cassani K. & $0.04 \%$ & $21.57 \%$ & 81 & Müller E. & $-10.62 \%$ *** & $32.01 \%$ \\
\hline 34 & Tanner E. & $0.03 \%$ & $16.84 \%$ & 82 & Blum G. & $-12.29 \%$ *** & $18.19 \%$ \\
\hline 35 & Hoffmann A. & $-0.12 \%$ & $13.35 \%$ & 83 & Borgeaud $\mathrm{P}$. & $-14.05 \%$ *** & $26.03 \%$ \\
\hline 36 & Hänggi R. & $-0.43 \%$ & $18.38 \%$ & 84 & Rauh M. & $-14.12 \%$ *** & $20.60 \%$ \\
\hline 37 & de Pury D. & $-0.72 \%$ & $22.97 \%$ & 85 & Goetz H. & $-14.28 \%$ *** & $22.38 \%$ \\
\hline 38 & Loepfe O. & $-0.81 \%$ & $17.41 \%$ & 86 & Amstutz M. & $-14.87 \%$ *** & $25.54 \%$ \\
\hline
\end{tabular}




\begin{tabular}{|c|c|c|c|c|c|c|c|}
\hline 39 & Maucher $\mathrm{H}$. & $-0.97 \%$ & $24.65 \%$ & 87 & Spälti P. & $-15.06 \%$ *** & $33.11 \%$ \\
\hline 40 & Leutwiler F. & $-1.12 \%$ & $17.32 \%$ & 88 & Spoerry V. & $-15.29 \%$ *** & $32.07 \%$ \\
\hline 41 & Feller K. & $-1.16 \%{ }^{*}$ & $18.79 \%$ & 89 & Schneider-Ammann J. & $-15.41 \%$ *** & $35.57 \%$ \\
\hline 42 & Jetzer A. & $-1.20 \% \%^{*}$ & $20.99 \%$ & 90 & Binkert $\mathrm{P}$. & $-16.00 \%$ *** & $21.35 \%$ \\
\hline 43 & Chaudet M. & $-1.41 \%$ ** & $16.13 \%$ & 91 & Mühlemann L. & $-16.00 \%$ *** & $30.86 \%$ \\
\hline 44 & Meier $\mathrm{H}$. & $-1.62 \%$ ** & $13.48 \%$ & 92 & Schmidheiny $\mathrm{T}$. & $-16.36 \%$ *** & $24.67 \%$ \\
\hline 45 & Bühler U. & $-1.76 \%$ ** & $23.89 \%$ & 93 & Honegger E. & $-18.85 \%$ *** & $34.79 \%$ \\
\hline 46 & Bechtler T. & $-1.82 \% \%^{\star \star \star}$ & $17.36 \%$ & 94 & Stähelin G. & $-19.86 \%$ *** & $33.53 \%$ \\
\hline 47 & Haerri H. & $-1.89 \%{ }^{* * \star}$ & $13.96 \%$ & 95 & Hentsch B. & $-28.04 \%$ *** & $43.00 \%$ \\
\hline 48 & Grete U. & $-2.37 \%$ \%** & $24.73 \%$ & & & & \\
\hline
\end{tabular}

Wie aus Tabelle 1 ersichtlich ist, differieren die Resultate sehr stark: Sie reichen von $+8.93 \%$ bis $-28.04 \%$ pro Jahr. Nur 34 Personen haben eine bessere Rendite als der Gesamtmarkt erreicht; im Schnitt beträgt sie $-3.7 \%$, während die Medianrendite bei $-2.37 \%$ liegt. Diese vom SPI stark abweichende Überrendite erklärt sich einerseits dadurch, dass die Verwaltungsratsmandate gleich gewichtet wurden. Weiter ist möglich, dass ein Selection Bias besteht: Es wurden nur Verwaltungsräte mit mindestens zwei Verwaltungsratsmandaten in SMI Firmen berücksichtigt. Es besteht die Möglichkeit, dass die in der Stichprobe nicht enthaltenen Personen mit nur einem Verwaltungsratsmandat besser abschneiden. Eine dritte Erklärung besteht darin, dass Firmen mit unterdurchschnittlicher Aktienkursentwicklung mehr Verwaltungsräte haben und möglicherweise auch benötigen, was dazu führt, dass sich in der vorliegenden Stichprobe mehr Verwaltungsräte von „schlechten“ als von „guten“ Firmen finden. Die relativ tiefen Volatilitäten sind darauf zurückzuführen, dass bei Zwischenperioden ohne Verwaltungsratsmandat eine Überrendite von null angenommen wurde.

Mit einem t-Test wurde untersucht, ob die Abweichungen vom Benchmark signifikant sind. Auf einem Signifikanzniveau von 95\% ist dies für 23 überdurchschnittliche Verwaltungsräte der Fall, während 53 Verwaltungsräte signifikant negative Abweichungen vom SPI zeigen.

Im nächsten Schritt wird auch das Risiko in die Analyse einbezogen. Für die risikoadjustierte Performancemessung wurden verschiedene Verfahren entwickelt. Zu den gebräuchlichsten gehören das SHARPE Ratio und JENSEN's Alpha. Währenddem für das SHARPE Ratio das Gesamtrisiko eines Portfolios von Bedeutung ist, wird beim JENSEN's Alpha nur das systematische Risiko relativ zu einem Marktportfolio analysiert. In beiden Fällen der rendite- und risikoorientierten Performancemessung wird die Rendite von Perioden ohne Mandatstätigkeit jeweils aufgrund des risikolosen Zinssatzes berechnet. Begründung dafür sind die Risiken, die mit einem Verwaltungsratsmandat verknüpft sind: Eine Aktienrisikoprämie ist nur für Personen gerechtfertigt, welche auch effektiv Risiken im Zusammenhang mit der Übernahme eines Mandates tragen.

\section{JENSEN's Alpha}

JENSEN's Alpha nach JENSEN (1968) misst die Renditedifferenz zwischen der tatsächlichen Rendite eines Portfolios und jener Rendite, welche aufgrund des systematischen Risikofaktors des Portfolios (Beta) erforderlich wäre. Ein Alpha grösser als Null wird als positive risikoadjustierte Performance interpretiert. Das Alpha wird als Achsenabschnitt der Zeitreihenregression 
$\mathrm{r}_{\mathrm{Pft}}-\mathrm{r}_{\mathrm{ft}}=\alpha+\beta\left(\mathrm{r}_{\mathrm{Mt}}-\mathrm{r}_{\mathrm{ft}}\right)+\varepsilon_{\mathrm{t}}$

ermittelt,

wobei: $\quad \mathrm{r}_{\mathrm{Pft}}$ : stetige Portfoliorendite für Periode $\mathrm{t}$;

$\mathrm{r}_{\mathrm{ft}}$ : risikoloser Zinssatz für Periode $\mathrm{t}$;

$\alpha$ : JENSEN's Alpha

$\beta$ : Beta

$\mathrm{r}_{\mathrm{Mt}}$ : stetige Benchmarkrendite für Periode $\mathrm{t}$;

$\varepsilon_{\mathrm{t}}$ : Residuum.

Für die Berechnung werden auch hier stetige Portfoliorenditen für die Periode von einer Woche verwendet. Die resultierenden Achsenabschnitte werden ebenso wie die Standardabweichungen auf Jahresbasis umgerechnet. Als Benchmark wird der Swiss Performance Index verwendet. Die unterschiedliche Branchenentwicklung ist hier weniger problematisch als bei der Berechnung der relativen Renditen, weil Verwaltungsratsmitglieder, die in Branchen mit einer überdurchschnittlichen Performance vertreten sind, tendenziell auch ein entsprechend höheres Portfoliobeta haben.

\section{SHARPE Ratio}

Das SHARPE Ratio oder Reward-to-Volatility Ratio nach SHARPE (1966) setzt die durchschnittliche Überrendite eines Portfolios gegenüber dem risikolosen Zinssatz $\left(r_{P f}-r_{f}\right)$ ins Verhältnis zum Risiko des Portfolios, gemessen mit der Volatilität der Portfoliorendite $\left(\sigma_{\mathrm{p}}\right)$ :

$\mathrm{S}_{\mathrm{Pf}}=\frac{\mathrm{r}_{\mathrm{Pf}}-\mathrm{r}_{\mathrm{f}}}{\sigma_{\mathrm{Pf}}}$

Das SHARPE Ratio eines Portfolios ist ein Mass für die Überrendite pro Einheit Risiko. Der Vorteil dieses Masses ist die Berechenbarkeit unabhängig von einem Benchmark. Bei der Interpretation der Ergebnisse muss berücksichtigt werden, dass das SHARPE Ratio Verwaltungsräte bevorteilt, die grössere Mandatsportfolios halten, da durch den Diversifikationseffekt die Volatilität mit der Anzahl Aktien im Portfolio tendenziell sinkt.

Mittels einer von JOBSON und KORKIE (1981) entwickelte Testgrösse wird untersucht, ob sich die SHARPE Ratios signifikant vom Median SHARPE Ratio unterscheiden. Sie erlaubt einen Vergleich zwischen zwei verschiedenen Portfolios, wobei die Nullhypothese besagt, dass die Differenz zwischen zwei SHARPE Ratios Null ist. Die Testgrösse ist approximativ standardnormalverteilt und wird nach folgender Formel berechnet:

$\mathrm{Z}_{\mathrm{PF}}=\frac{\overline{\mathrm{r}}_{1} \cdot \sigma_{2}-\overline{\mathrm{r}}_{2} \cdot \sigma_{1}}{\sqrt{\theta}}$

mit:

$\theta=\frac{1}{\mathrm{~T}}\left[2 \cdot \sigma_{1}^{2} \cdot \sigma_{2}^{2}-2 \cdot \sigma_{1}^{2} \cdot \sigma_{2}^{2} \cdot \rho+\frac{1}{2} \cdot \bar{r}_{1}^{2} \cdot \sigma_{2}^{2}+\frac{1}{2} \cdot \bar{r}_{2}^{2} \cdot \sigma_{1}^{2}-\frac{\bar{r}_{1} \cdot \bar{r}_{2}}{2 \cdot \sigma_{1} \cdot \sigma_{2}}\left(\rho^{2} \cdot \sigma_{1}^{2} \cdot \sigma_{2}^{2}-\sigma_{1}^{2} \cdot \sigma_{2}^{2}\right)\right]$

wobei: 
$\overline{\mathrm{r}}_{\mathrm{i}}=$ durchschnittliche Überrendite des jeweiligen Portfolios

$\sigma_{\mathrm{i}}=$ Standardabweichung des jeweiligen Portfolios

$\rho=$ Korrelationskoeffizient zwischen den Überschussrenditen der Portfolios

$\mathrm{T}=$ Anzahl Beobachtungen

\section{Appraisal Ratio}

Das Appraisal Ratio oder TREYNOR/BLACK Ratio nach TREYNOR und BLACK (1973) setzt das Alpha ins Verhältnis zum unsystematischen Risiko:

Appraisal Ratio = JENSEN`s Alpha / Tracking Error

Der Tracking Error wird als Standardabweichung des Störterms $\left(\varepsilon_{\mathrm{PFt}}\right)$ aus Gleichung 2 berechnet.

Tabelle 3 zeigt die Rangliste geordnet nach den JENSEN's Alphas mit den SHARPE Ratios und den Appraisal Ratios (AR) der individuellen Portfolios der Verwaltungsräte.

Tabelle 2: JENSEN's Alphas, SHARPE Ratios und Apraisal Ratios der Verwaltungsratsportfolios

\begin{tabular}{|c|c|c|c|c|c|c|c|c|c|}
\hline Rang & Name & $\begin{array}{c}\text { JENSEN's } \\
\text { Alpha }\end{array}$ & $\begin{array}{c}\text { SHARPE } \\
\text { Ratio } \\
\end{array}$ & AR & Rang & Name & $\begin{array}{c}\text { JENSEN's } \\
\text { Alpha }\end{array}$ & $\begin{array}{c}\text { SHARPE } \\
\text { Ratio }\end{array}$ & AR \\
\hline 1 & Sarasin B. & $9.30 \%$ & $0.65^{\star \star \star}$ & 0.35 & 49 & Zbinden $\mathrm{P}$. & $-1.13 \%$ & $0.07^{\star \star \star}$ & -0.03 \\
\hline 2 & Sigg $\mathrm{H}$. & $9.06 \%$ & $0.74^{\star * *}$ & 0.43 & 50 & Jetzer A. & $-1.19 \%$ & $0.47^{\star \star \star}$ & -0.09 \\
\hline 3 & Bodmer A. & $8.69 \%{ }^{*}$ & $0.79^{\star \star \star}$ & 0.59 & 51 & Genillard R. & $-1.40 \%$ & $0.49^{\star \star \star}$ & -0.09 \\
\hline 4 & Blocher C. & $8.68 \%$ ** & $0.92^{\star \star \star}$ & 0.64 & 52 & Dunkel A. & $-1.61 \%$ & $0.48^{\star \star *}$ & -0.13 \\
\hline 5 & Küpfer P. & $8.49 \%$ & $0.64^{\star \star \star}$ & 0.48 & 53 & Zumstein J. & $-1.63 \%$ & $0.02^{\star \star \star}$ & -0.18 \\
\hline 6 & Languetin P. & $6.71 \%$ & $0.63^{\star \star \star}$ & 0.32 & 54 & Pozzi A. & $-1.74 \%$ & 0.29 & -0.13 \\
\hline 7 & Jucker H. & $6.69 \%{ }^{*}$ & $0.80^{\star \star \star}$ & 0.54 & 55 & Landolt P. & $-1.90 \%$ & 0.40 & -0.14 \\
\hline 8 & Gasser R. & $6.13 \%$ & $0.69^{\star \star \star}$ & 0.38 & 56 & de Pury D. & $-2.36 \%$ & $0.45^{\star \star \star}$ & -0.17 \\
\hline 9 & Junger M. & $5.86 \%$ & $0.63^{\star \star \star}$ & 0.27 & 57 & Kielholz W. & $-2.49 \%$ & $0.04^{\star \star \star}$ & -0.21 \\
\hline 10 & Maucher $\mathrm{H}$. & $5.51 \%$ & $0.53^{\star \star \star}$ & 0.25 & 58 & Marchionne S. & $-2.77 \%$ & $-0.04^{\star * *}$ & -0.25 \\
\hline 11 & Gugler A. & $5.17 \%$ & $0.77^{\star \star \star}$ & 0.45 & 59 & Schweizer R. & $-3.13 \%$ & 0.31 & -0.15 \\
\hline 12 & Chaudet M. & $4.72 \%$ & $0.60^{\star \star \star}$ & 0.32 & 60 & Cappis M. & $-3.32 \%$ & $0.20^{\star \star \star}$ & -0.20 \\
\hline 13 & Schäuble R. & $4.69 \%$ & $0.66^{\star * *}$ & 0.28 & 61 & Senn N. & $-3.70 \%$ & $0.24^{\star *}$ & -0.15 \\
\hline 14 & Grete U. & $4.16 \%$ & 0.43 & 0.19 & 62 & von Finck $A$. & $-3.86 \%$ & $0.18^{\star \star \star}$ & -0.24 \\
\hline 15 & Galliker F. & $4.09 \%$ & $0.61^{* * *}$ & 0.43 & 63 & Vasella D. & $-3.98 \%$ & 0.33 & -0.35 \\
\hline 16 & Studer R. & $4.01 \%$ & $0.58^{\star \star \star}$ & 0.22 & 64 & Hoffmann A. & $-4.75 \%$ & $0.02^{\star \star \star}$ & -0.42 \\
\hline 17 & Bremi U. & $3.98 \%$ & $0.58^{\star \star \star}$ & 0.21 & 65 & Kündig M. & $-4.93 \%$ & $0.22^{\star \star \star}$ & -0.35 \\
\hline 18 & Loepfe O. & $3.95 \%$ & $0.65^{\star \star \star}$ & 0.29 & 66 & Lippuner H. & $-5.42 \%$ & 0.34 & -0.40 \\
\hline 19 & Muller G. & $3.24 \%$ & $0.44^{*}$ & 0.14 & 67 & Jeker R. & $-5.44 \%$ & $0.15^{\star \star \star}$ & -0.34 \\
\hline 20 & Diehl W. & $3.09 \%$ & $0.44^{*}$ & 0.26 & 68 & Tschopp T. & $-5.62 \%$ & $0.20^{\star \star \star}$ & -0.38 \\
\hline 21 & Moret M. & $3.02 \%$ & $0.49^{* *}$ & 0.28 & 69 & Leuenberger $\mathrm{A}$. & $-6.53 \%$ & $0.14^{\star \star \star}$ & -0.32 \\
\hline 22 & Cassani K. & $2.92 \%$ & $0.65^{\star \star \star}$ & 0.20 & 70 & Bodmer $\mathrm{H}$. & $-7.29 \%$ & $0.22^{* \star \star}$ & -0.38 \\
\hline 23 & Albers U. & $2.90 \%$ & $0.58^{\star \star \star}$ & 0.15 & 71 & Kissling W. & $-7.60 \%$ & $0.05^{\star \star \star}$ & -0.29 \\
\hline 24 & Milliet F. & $2.80 \%$ & $0.48^{\star *}$ & 0.24 & 72 & Schmidheiny J. & $-7.66 \%$ & $0.06^{\star \star \star}$ & -0.47 \\
\hline 25 & Leutwiler F. & $2.71 \%$ & $0.63^{\star \star \star}$ & 0.22 & 73 & Brabeck P. & $-7.72 \%{ }^{*}$ & $0.04^{\star \star \star}$ & -0.52 \\
\hline 26 & Gut R. & $2.70 \%$ & $0.71^{\star \star \star}$ & 0.23 & 74 & Blum G. & $-8.30 \% *$ & $-0.06^{\star \star *}$ & -0.57 \\
\hline 27 & Frehner W. & $2.46 \%$ & $0.67^{\star * \star}$ & 0.20 & 75 & Hüppi R. & $-8.31 \%$ & $0.15^{\star \star \star}$ & -0.42 \\
\hline 28 & Ruepp R. & $2.35 \%$ & $0.51^{\star \star \star}$ & 0.18 & 76 & Domeniconi R. & $-8.44 \%$ & $0.00^{\star \star \star}$ & -0.48 \\
\hline 29 & Krauer A. & $2.31 \%$ & $0.68^{\star * *}$ & 0.19 & 77 & Humer F. & $-9.49 \%$ ** & $-0.11^{\star * *}$ & -0.71 \\
\hline 30 & Feller K. & $2.30 \%$ & $0.53^{\star \star \star}$ & 0.15 & 78 & Müller E. & $-9.64 \%$ & $0.02^{\star \star \star}$ & -0.34 \\
\hline
\end{tabular}




\begin{tabular}{|c|c|c|c|c|c|c|c|c|c|}
\hline 31 & Gisling J. & $1.99 \%$ & 0.33 & 0.09 & 79 & Binkert $P$. & $-9.69 \%$ & $-0.18^{\star \star \star}$ & -0.49 \\
\hline 32 & Gerber F. & $1.98 \%$ & $0.62^{\star \star \star}$ & 0.15 & 80 & Lehn J. & $-9.74 \% *$ & $-0.08^{\star \star *}$ & -0.55 \\
\hline 33 & Schmidheiny S. & $1.88 \%$ & $0.61^{\star \star \star}$ & 0.13 & 81 & Meyer R. & $-9.98 \% *$ & $0.03^{\star \star \star}$ & -0.57 \\
\hline 34 & Ebner M. & $1.72 \%$ & $0.63^{\star \star \star}$ & 0.13 & 82 & Goetz H. & $-10.46 \%{ }^{*}$ & $-0.14^{\star \star *}$ & -0.54 \\
\hline 35 & Bühler U. & $1.70 \%$ & $0.51^{\star \star \star}$ & 0.09 & 83 & Höhler G. & $-12.00 \%$ ** & $-0.34^{* * *}$ & -0.83 \\
\hline 36 & Haerri H. & $1.50 \%$ & $0.23^{*}$ & 0.11 & 84 & Schneider-Ammann J. & $-14.03 \%$ & $-0.12^{\star \star \star}$ & -0.43 \\
\hline 37 & Tanner E. & $1.50 \%$ & $0.47^{\star *}$ & 0.10 & 85 & Amstutz M. & $-14.20 \%$ ** & $-0.15^{\star \star \star}$ & -0.67 \\
\hline 38 & de Kalbermatten B. & $1.30 \%$ & 0.38 & 0.08 & 86 & van Wachem L. & $-14.48 \%$ ** & $-0.05^{\star \star \star}$ & -0.81 \\
\hline 39 & Ackermann J. & $1.16 \%$ & 0.34 & 0.05 & 87 & Borgeaud P. & $-14.90 \%$ ** & $-0.11^{\star \star *}$ & -0.75 \\
\hline 40 & Doerig $\mathrm{H}$. & $1.15 \%$ & $0.53^{* \star *}$ & 0.09 & 88 & Schmidheiny $\mathrm{T}$. & $-15.11 \%$ ** & $-0.21^{\star \star \star}$ & -0.76 \\
\hline 41 & Grether E. & $1.04 \%$ & $0.46^{\star *}$ & 0.05 & 89 & Spoerry V. & $-16.73 \%$ ** & $-0.13^{\star * *}$ & -0.64 \\
\hline 42 & Meier $\mathrm{H}$. & $0.82 \%$ & 0.33 & 0.07 & 90 & Spälti P. & $-17.83 \%$ ** & $-0.12^{\star \star *}$ & -0.66 \\
\hline 43 & Angst C. & $0.47 \%$ & $0.18^{* * *}$ & 0.03 & 91 & Rauh M. & $-18.12 \%$ ** & $-0.67^{* \star *}$ & -0.93 \\
\hline 44 & BechtlerT. & $0.33 \%$ & $0.54^{\star \star \star}$ & 0.03 & 92 & Stähelin G. & $-18.75 \%$ ** & $-0.26^{\star \star *}$ & -0.62 \\
\hline 45 & Böckli P. & $0.24 \%$ & $0.61^{\star \star *}$ & 0.02 & 93 & Mühlemann L. & $-19.04 \%$ ** & $-0.32^{\star \star *}$ & -0.69 \\
\hline 46 & Hänggi R. & $-0.43 \%$ & 0.40 & -0.03 & 94 & Honegger $\mathrm{E}$. & $-19.50 \%{ }^{*}$ & $-0.32^{\star \star \star}$ & -0.60 \\
\hline 47 & von der Crone H. & $-0.77 \%$ & $0.24^{* *}$ & -0.05 & 95 & Hentsch B. & $-25.31 \%{ }^{\star}$ & $-0.39^{* * *}$ & -0.62 \\
\hline 48 & Müller-Berghof B. & $-0.94 \%$ & 0.37 & -0.06 & & SPI & & 0.69 & \\
\hline
\end{tabular}

Rund die Hälfte aller Personen weist ein positives Alpha aus. Auf dem 95\% Signifikanzniveau weist nur ein einziger Verwaltungsrat eine Überperformance auf: Christoph Blocher. Das bekannte Bild bei der negativen Performance wiederholt sich aber auch hier: für elf Personen ergibt die Analyse auf diesem Niveau ein statistisch signifikant negatives Alpha. Es fällt allerdings auf, dass die Resultate von Eric Honegger und Benedict Hentsch, im Gegensatz zur rein renditeorientierten Performanceanalyse, bei dieser Betrachtungsweise nur auf einem 10\% Niveau signifikant sind. Der Grund dafür liegt in der vergleichsweise hohen spezifischen Volatilität der Mandatsportfolios dieser Personen.

Die SHARPE Ratios wurden darauf getestet, ob sie vom Median SHARPE Ratio von Heini Lippuner (0.34) verschieden sind. Die Nullhypothese, dass das SHARPE Ratio gleich dem Median SHARPE Ratio ist, wird für 74 Verwaltungsräte auf dem Signifikanzniveau von $1 \%$ verworfen. Durch den zusätzlichen Einbezug der Volatilität verschlechtert sich die Performance der Verwaltungsratsportfolios im Vergleich zum Benchmark, was jedoch eine unfaire Gegenüberstellung bedeutet, da der SPI viel breiter diversifiziert ist. Dies verdeutlicht die Tatsache, dass nur 14\% der Portfolios eine tiefere Volatilität haben als der SPI. Insgesamt resultieren nur sechs Verwaltungsräte mit einem höheren SHARPE Ratio als der Gesamtmarktindex.

Die Berücksichtigung des unsystematischen Risikos über das Appraisal Ratio führt dazu, dass Verwaltungsräte der grossen, den SPI prägenden Firmen tendenziell besser abschneiden als die übrigen.

Mit einem SPEARMAN Rangkorrelationstest nach KANJI (1999) wurde getestet, ob die unterschiedlichen Rangfolgen nach Überrenditen, JENSEN's Alphas und SHARPE Ratios korreliert sind. Für die Beziehung zwischen den Rängen nach Überrenditen und denjenigen nach JENSEN's Alphas ergibt sich dabei ein Wert von -7.60 und für die Beziehung zwischen der Rangierung nach JENSEN's Alphas und derjendigen nach SHARPE Ratios ein solcher von -8.94. Dies bedeutet, dass die Nullhypothese „,keine Korrelation zwischen den Rängen“ in beiden Fällen auf 
einem Signifikanzniveau von $1 \%$ verworfen werden kann. Obwohl die Überrenditen das Risiko überhaupt nicht berücksichtigen und bei JENSEN's Alpha und SHARPE Ratio unterschiedliche Risikodefinitionen zu Grunde liegen, besteht also ein ausgeprägter Zusammenhang zwischen den Ranglisten.

Insgesamt ist das Bild von Verwaltungsräten geprägt, die bedeutend schlechter abschneiden als der Markt. Eine Erklärung dafür ist die mangelnde Diversifikation der jeweiligen Portfolios und die daraus resultierende vergleichsweise hohe Volatilität. Die negative Performance lässt sich in vielen Fällen auch mit einer stark negativen Rendite einer Unternehmung in einer einzelnen Periode begründen (beispielsweise Swissair 2001, Centerpulse respektive Sulzer Medica 2001). Statistisch signifikant negative Resultate aufgrund einmaliger Unternehmensnotlagen sind nicht unbedingt geeignet, den Einfluss von Verwaltungsratsmitgliedern auf die Performance zu quantifizieren. Die Resultate zeigen, dass entweder die Unternehmensperformance nur in einem beschränkten Ausmass durch die einzelnen Verwaltungsräte bestimmt wird, da sich für niemanden anhand mehrerer Berechnungsverfahren eine statistisch signifikante Überperformance ergibt, oder dass keine Verwaltungsräte mit einer systematischen Outperformance der Aktie assoziiert werden können. Eine weitere mögliche Erklärung besteht darin, dass Firmen mit einer unterdurchschnittlichen Aktienkursentwicklung mehr Verwaltungsräte benötigen. In der vorliegenden Analyse äussert sich dies in einer unterdurchschnittlichen Performance der Personen, weil in der Stichprobe somit mehr Verwaltungsräte von „schlechten“ als von ,guten“ Firmen zu finden sind.

Die vorhergehenden Portfolioperformanceanalysen bewerten und vergleichen die Leistung von Verwaltungsräten über den Beobachtungszeitraum. Die Portfoliobetrachtung hat insbesondere den Vorteil, dass die Resultate auch mit anderen Kapitalanlagen, Fonds oder Benchmarks verglichen werden können. Allerdings ist die Aussagekraft in Bezug auf die effektive Leistung eines Verwaltungsrates, trotz Risikoadjustierung und relativer Renditeberechnung, mit gewissen Einschränkungen verbunden, weil die Performance zu einem wesentlichen Teil von der jeweiligen Portfoliozusammensetzung abhängt. So beeinflusst eine titelspezifische Rendite in einer Periode mit nur einem einzigen Mandat die „Verwaltungsratsrendite“ stärker als in Perioden mit mehreren Mandaten. Dies hat zwar seine Berechtigung in Bezug auf die Portfoliobetrachtungsweise, ist aber für die Leistungsbeurteilung nicht optimal, da die Hauptaufgabe eines Verwaltungsrates die Aufsicht einer Gesellschaft und nicht das Verwalten seines Mandatsportefeuilles ist. Aus diesem Grund wird im Folgenden eine alternative Methodik zur Performanceberechnung und -analyse präsentiert.

\subsection{Durchschnittsperformanceanalysen und Persistenz}

Für die Durchschnittsperformanceanalysen werden die verschiedenen Mandate und entsprechenden Renditen im Gegensatz zu Abschnitt 4.1 nicht mehr aufgrund der zeitlichen Abfolge aggregiert, sondern die Unternehmensrenditen werden unabhängig vom Zeitpunkt und allfällig parallel laufenden Mandaten betrachtet. In diesem Sinn sind diese Verfahren eine Durchschnittsbetrachtung über alle Mandate. Der Vorteil davon ist, dass zeitlich wechselnde Portfoliozusammensetzungen keine Rolle mehr spielen und sämtliche Mandatsjahre gleich gewichtet werden; gleichzeitig fallen Perioden ohne Mandatstätigkeit weg.

Nachteilig wirkt sich jedoch aus, dass das Risiko nicht mehr korrekt einbezogen werden kann. Zwar könnte auch hier die Volatilität mitberücksichtigt werden, da aber die zeitliche Abfolge in den Verwaltungsratsportfolios nicht mehr gegeben ist, die Volatilität aber wesentlich von der 
jeweiligen Marktsituation abhängt, würde eine Risikoadjustierung zu fehlerhaften Interpretationen führen.

Für die Berechnung der durchschnittlichen Rendite spielt die jeweilige Mandatsdauer eine wichtige Rolle: Um sicherzustellen, dass eine lange Verwaltungsratstätigkeit im Vergleich zu einer kurzen angemessen gewichtet wird, werden die durchschnittlichen Renditen je Mandat mit der Dauer der Tätigkeit beim Unternehmen gewichtet.

Es wird wiederum die relative Rendite gegenüber dem SPI als Benchmark ausgewiesen. Absolute Renditen wären irreführend, da nicht alle Verwaltungsräte über den ganzen Zeitraum Mandate innehatten. Zweck dieser Art der Performanceberechnung ist eine Erhöhung der Aussagekraft bezüglich der effektiven Leistung eines Verwaltungsrates. Die erzielte Rendite steht in einem engeren Zusammenhang mit dem Wirken des Verwaltungsrates.

Eine wichtige Fragestellung betrifft die Nachhaltigkeit der ermittelten Performance. Es interessiert, ob die „guten“ Verwaltungsräte auch in Bezug auf ein Zeitintervall von fünf Jahren besser abschneiden als „,schlechte“ Verwaltungsräte. Dies wäre ein Anzeichen dafür, dass eine Überrendite von der Person eines Verwaltungsrates beeinflusst wird und weniger von anderen Faktoren abhängt.

Über die Persistenz in der Performance gibt

Tabelle 3 Auskunft. Die zehnjährige Zeitreihe wurde in zwei Subperioden von 1992 bis 1996 und von 1997 bis 2001 unterteilt und wiederum die Rendite relativ zum SPI berechnet. Die Rangierung erfolgt nach der Durchschnittsrendite für die gesamte Periode. Der Mittelwert über die gesamte Periode beträgt $-4.99 \%$, für die erste Subperiode $-4.03 \%$ und für die zweite $-6.68 \%$. Auch hier können die grossen Abweichungen vom SPI auf die Gleichgewichtung der Mandate und auf des Fehlen der Verwaltungsräte mit nur einem Mandat zurückgeführt werden. Die im Vergleich zu Tabelle 1 noch stärker negativen Durchschnittsresultate erklären sich dadurch, dass hier die Jahre ohne Mandatstätigkeit nicht in der Stichprobe enthalten sind. Vergleicht man die Ranglisten in den Tabellen 1 und 3, so ergibt der SPEARMAN Rangkorrelationstest nach KANJI (1999) einen z-Wert von -8.79 was auf einem Signifikanzniveau von $1 \%$ auf Korrelation in den Ranglisten hinweist. Die unterschiedlichen Berechnungsmethoden haben also nur einen beschränkten Einfluss auf die Rangfolge der Verwaltungsräte.

Tabelle 3: Performance in den Subperioden

\begin{tabular}{|c|c|c|c|c|c|c|c|c|c|}
\hline \multirow[b]{2}{*}{ Rang } & \multirow[b]{2}{*}{ Name } & \multicolumn{3}{|c|}{ Überrendite } & \multirow[b]{2}{*}{ Rang } & \multirow[b]{2}{*}{ Name } & \multicolumn{3}{|c|}{ Überrendite } \\
\hline & & $92-01$ & 92-96 & $97-01$ & & & $92-01$ & $92-96$ & $97-01$ \\
\hline 1 & Kielholz W. & $13.48 \% \%^{\star \star \star}$ & - & $13.48 \% \%^{\star \star \star}$ & 49 & Hänggi R. & $-3.10 \%^{*}$ & $2.80 \% \%^{\star \star \star}$ & $-4.41 \%$ \\
\hline 2 & Diehl W. & $7.82 \%$ *** & $7.82 \%$ *** & - & 50 & Haerri $\mathrm{H}$. & $-3.18 \% *$ & $-3.18 \%$ & - \\
\hline 3 & Schäuble R. & $7.62 \%$ *** & $-3.66 \%$ & $15.13 \% \%^{\star \star \star}$ & 51 & Bodmer $\mathrm{H}$. & $-3.70 \%$ & $-2.11 \%{ }^{\star \star \star}$ & $-8.47 \%$ \\
\hline 4 & Sarasin B. & $7.59 \% \%^{\star \star \star}$ & $0.84 \%$ *** & $19.74 \%^{* \star *}$ & 52 & Müller-Berghof $\mathrm{B}$. & $-3.74 \%$ & $1.60 \%$ *** & $-27.77 \%$ *** \\
\hline 5 & Marchionne S. & $7.11 \% \%^{* * *}$ & - & $7.11 \%$ *** & 53 & Lippuner $\mathrm{H}$. & $-3.85 \%$ & $-10.41 \%$ *** & $2.71 \%^{\star * *}$ \\
\hline 6 & Muller G. & $6.59 \%$ *** & $4.96 \%^{* \star *}$ & $7.28 \% \%^{* \star \star}$ & 54 & Leutwiler F. & $-3.94 \%$ & $-5.91 \%$ *** & $8.87 \%$ *** \\
\hline 7 & Gasser R. & $6.08 \% \%^{* \star \star}$ & $10.27 \%$ *** & $3.99 \% * * *$ & 55 & von Finck $A$. & $-4.33 \%$ & $-1.72 \%$ *** & $-5.76 \%$ \\
\hline 8 & Brabeck P. & $4.41 \% \%^{* * *}$ & - & $4.41 \% \%^{\star \star \star}$ & 56 & Meyer R. & $-4.34 \%$ & $-11.56 \%$ *** & $-1.63 \% \%^{\star \star \star}$ \\
\hline 9 & Chaudet M. & $3.32 \% \%^{\star \star \star}$ & $-2.02 \% \%^{\star \star \star}$ & $7.18 \% \%^{\star \star \star}$ & 57 & Doerig $\mathrm{H}$. & $-4.57 \%$ & $-0.73 \%^{* \star *}$ & $-10.32 \%$ ** \\
\hline 10 & Hoffmann A. & $3.31 \%$ *** & - & $3.31 \% * * *$ & 58 & Tschopp T. & $-4.57 \%$ & $-5.93 \% * * *$ & $-4.11 \%$ \\
\hline 11 & Languetin $\mathrm{P}$. & $3.25 \%$ *** & $1.58 \%$ *** & $7.27 \%$ *** & 59 & von der Crone $\mathrm{H}$. & $-5.58 \%$ & $-0.15 \%$ *** & $-17.91 \%$ *** \\
\hline 12 & Sigg $\mathrm{H}$. & $3.25 \% \%^{* * *}$ & $-5.07 \%{ }^{*}$ & $15.72 \%^{\star \star \star}$ & 60 & van Wachem L. & $-5.77 \%$ & $-3.75 \%$ & $-6.89 \%$ \\
\hline
\end{tabular}




\begin{tabular}{|c|c|c|c|c|c|c|c|c|c|}
\hline 13 & Bremi U. & $3.19 \%$ *** & $-0.75 \%$ *** & $7.39 \%$ *** & 61 & Ruepp R. & $-5.97 \%$ & $-8.42 \%{ }^{* * *}$ & $-2.48 \% \%^{\star * *}$ \\
\hline 14 & Junger M. & $2.93 \%{ }^{* \star *}$ & $-5.62 \%{ }^{\star \star *}$ & $14.90 \%$ *** & 62 & Cappis M. & $-6.25 \%$ & $-7.65 \%$ \%** & $-4.37 \%$ \\
\hline 15 & Dunkel A. & $2.77 \%$ \%** & $-7.34 \%$ *** & $6.81 \%{ }^{* * *}$ & 63 & Ackermann J. & $-6.59 \%$ & $2.32 \%$ *** & $-22.92 \%$ *** \\
\hline 16 & Albers U. & $2.68 \%{ }^{\star \star \star}$ & $0.79 \%$ \%** & $6.46 \% \%^{\star \star \star}$ & 64 & Kündig M. & $-6.91 \% *$ & $-6.68 \%$ *** & $-7.15 \%$ \\
\hline 17 & Tanner E. & $2.64 \%{ }^{* \star *}$ & $-1.17 \%$ *** & $3.68 \% \%^{* \star \star}$ & 65 & Höhler G. & $-7.00 \%{ }^{\star *}$ & - & $-7.00 \%$ \\
\hline 18 & Vasella D. & $2.57 \%$ *** & - & $2.57 \% \%^{* * *}$ & 66 & Milliet F. & $-7.31 \%$ ** & $-6.60 \% \%^{* \star *}$ & $-15.18 \% \%^{\star \star *}$ \\
\hline 19 & Gut $\mathrm{R}$. & $2.54 \%$ *** & $0.64 \%$ *** & $7.12 \%$ *** & 67 & Senn N. & 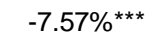 & $-9.65 \%$ *** & $-0.08 \%$ *** \\
\hline 20 & Landolt $\mathrm{P}$. & $1.86 \%$ *** & $-3.68 \%$ & $4.63 \% \%^{* \star *}$ & 68 & de Kalbermatten B. & $-7.68 \%$ *** & 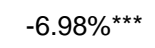 & $-9.51 \% *$ \\
\hline 21 & Krauer A. & $1.78 \%$ *** & $-3.92 \%$ & $8.44 \% * * *$ & 69 & Küpfer $P$. & $-7.96 \%$ *** & $-5.60 \%$ *** & $-9.40 \% *$ \\
\hline 22 & Genillard R. & $1.50 \%$ *** & $2.18 \%$ *** & $0.65 \% * * *$ & 70 & Pozzi A. & $-8.78 \%$ *** & $-8.57 \%$ *** & $-9.16 \%$ \\
\hline 23 & Grether E. & $1.45 \% \%^{\star \star \star}$ & $1.83 \% \%^{* * *}$ & $0.69 \%$ *** & 71 & Lehn J. & $-8.98 \%$ *** & $-3.77 \%$ & $-14.19 \%$ *** \\
\hline 24 & Jucker $\mathrm{H}$. & $0.74 \%$ *** & $0.66 \%$ *** & $1.15 \% \%^{\star \star \star}$ & 72 & Gisling J. & $-9.38 \%$ *** & $-12.34 \%$ *** & $-1.97 \%$ *** \\
\hline 25 & Studer R. & $0.70 \%$ *** & $-0.88 \%$ *** & $2.76 \% \%^{\star \star \star}$ & 73 & Jeker R. & $-9.79 \% \%^{\star \star \star}$ & $-2.30 \%$ *** & $-14.90 \%$ *** \\
\hline 26 & Moret M. & $0.61 \%$ *** & $0.61 \%$ *** & - & 74 & Schmidheiny J. & $-9.80 \%$ *** & $-4.10 \%$ & $-15.91 \%$ *** \\
\hline 27 & Blocher C. & $0.58 \%$ *** & $1.46 \%$ *** & $-0.60 \%$ *** & 75 & Spälti P. & $-10.16 \%$ *** & $-4.87 \%$ & $-16.52 \%$ *** \\
\hline 28 & Grete U. & $0.55 \%$ *** & $3.70 \%$ *** & $-7.83 \%$ & 76 & Zbinden $\mathrm{P}$. & $-10.43 \%$ *** & $-7.68 \%$ *** & $-17.31 \%$ *** \\
\hline 29 & Schweizer R. & $0.42 \%$ *** & $3.70 \%$ *** & $-3.94 \%{ }^{*}$ & 77 & Zumstein J. & $-10.68 \%$ *** & $-10.68 \%$ *** & - \\
\hline 30 & Schmidheiny S. & $0.25 \%$ *** & $-0.21 \%$ *** & $1.91 \%$ *** & 78 & Domeniconi R. & $-11.59 \%$ *** & $-4.84 \%$ & $-12.52 \%$ *** \\
\hline 31 & Gerber F. & $-0.03 \%$ *** & $2.20 \%$ *** & $-3.27 \%$ ** & 79 & Kissling W. & $-11.76 \%$ *** & $-1.12 \%$ *** & $-14.60 \%$ *** \\
\hline 32 & Cassani K. & $-0.28 \%$ *** & $-0.79 \%$ *** & $0.59 \% \%^{* * *}$ & 80 & Blum G. & $-11.94 \%$ *** & $-9.80 \% \%^{\star \star \star}$ & $-13.51 \%{ }^{* * *}$ \\
\hline 33 & Jetzer A. & $-0.36 \%{ }^{\star \star \star}$ & $-0.90 \%{ }^{* \star \star}$ & $0.82 \%^{* * *}$ & 81 & Borgeaud P. & $-12.35 \%^{* * *}$ & $-7.99 \% \%^{\star \star \star}$ & $-17.75 \%$ *** \\
\hline 34 & Galliker F. & $-0.56 \%$ *** & $-0.56 \%$ *** & - & 82 & Binkert $P$. & $-13.49 \%$ *** & $-6.96 \%$ *** & $-52.66 \%$ *** \\
\hline 35 & Feller K. & $-0.94 \%$ *** & $-6.08 \% \%^{\star \star \star}$ & $3.18 \% \%^{* * *}$ & 83 & Amstutz M. & $-14.05 \%$ *** & $-14.05 \%$ *** & $-14.05 \%$ *** \\
\hline 36 & Frehner W. & $-1.00 \%$ *** & $-0.90 \%$ *** & $-1.13 \%$ *** & 84 & Spoerry V. & $-14.18 \%$ *** & $-0.86 \%$ *** & $-30.16 \%$ *** \\
\hline 37 & de Pury D. & $-1.02 \%{ }^{* \star *}$ & $-1.59 \% \%^{* * *}$ & $-0.10 \%$ *** & 85 & Schneider-Am & $-14.51 \%$ *** & $-23.84 \%^{* * *}$ & $-5.18 \%$ \\
\hline 38 & Loepfe O. & $-1.03 \%$ *** & $-3.16 \%$ & $4.07 \%$ *** & 86 & Humer F. & $-16.82 \%$ *** & - & $-16.82 \%$ *** \\
\hline 39 & Bodmer A. & $-1.06 \%$ *** & $0.68 \% \%^{\star \star \star}$ & $-14.98 \%$ *** & 87 & Schmidheiny $\mathrm{T}$. & 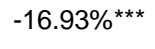 & $-3.16 \%$ & $-33.82 \% \%^{\star \star \star}$ \\
\hline 40 & Böckli P. & $-1.22 \%$ *** & $-7.63 \%^{\star \star \star}$ & $8.17 \% \%^{\star \star *}$ & 88 & Müller E. & $-17.33 \%$ *** & $-14.70 \% \%^{\star \star \star}$ & $-21.10 \%$ *** \\
\hline 41 & Hüppi R. & $-1.30 \%$ *** & $3.26 \%$ *** & $-4.34 \%$ & 89 & Goetz H. & $-17.67 \%$ *** & $-26.78 \%$ *** & $-6.66 \%$ \\
\hline 42 & Meier $\mathrm{H}$. & $-1.35 \% \%^{\star \star \star}$ & $-2.54 \% \%^{\star \star \star}$ & $-1.25 \%$ *** & 90 & Stähelin G. & 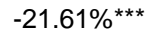 & $-7.85 \%$ *** & $-34.39 \% \%^{\star \star \star}$ \\
\hline 43 & Ebner M. & $-1.68 \%$ *** & $-2.46 \%$ *** & $-1.27 \%^{\star \star \star}$ & 91 & Hentsch B. & $-24.69 \%$ *** & $-1.34 \%^{\star * *}$ & $-41.37 \%$ *** \\
\hline 44 & Bühler U. & $-1.79 \% \%^{\star \star \star}$ & $-2.26 \%$ *** & $-0.29 \%$ *** & 92 & Leuenberger $\mathrm{A}$. & $-26.07 \% * \star \star *$ & $10.69 \%$ *** & $-43.03 \%$ *** \\
\hline 45 & Bechtler T. & $-2.23 \%$ *** & $-3.06 \%{ }^{*}$ & $-1.48 \%^{* * *}$ & 93 & Rauh M. & $-31.44 \%^{* * *}$ & - & $-31.44 \%$ *** \\
\hline 46 & Gugler A. & $-2.69 \%$ ** & $0.00 \% \%^{\star \star *}$ & $-7.73 \%$ & 94 & Mühlemann L. & $-35.60 \%$ *** & $-0.17 \%$ *** & $-42.04 \%$ *** \\
\hline 47 & Maucher $\mathrm{H}$. & $-3.10 \% *$ & $-4.33 \%$ & $-0.64 \%$ *** & 95 & Honegger $\mathrm{E}$. & $-49.40 \%$ *** & $-1.72 \%$ *** & $-61.32 \%$ *** \\
\hline $4 \varepsilon$ & Angst C. & $-3.10 \%$ * & $-3.10 \%$ * & - & & & & & \\
\hline
\end{tabular}

${ }^{* * * / * * *}$ bedeutet Signifikanz auf dem Niveau von $10 \% / 5 \% / 1 \%$.

Sämtliche Grössen sind annualisiert.

Rangierung gemäss Performance in der ersten Subperiode

Der SPEARMAN Rangkorrelationstest für die 81 Verwaltungsräte, welche in beiden Perioden aktiv waren, ergibt einen z-Wert von 0.34 , so dass die Nullhypothese „keine Rangkorrelation“ nicht verworfen werden kann. Eine Nachhaltigkeit in der Verwaltungsratsperformance kann auf diese Weise also nicht festgestellt werden.

Tabelle 4: Quartile für zwei Subperioden

\begin{tabular}{cccccc}
\hline & \multicolumn{4}{c}{ Rang 1992-1996 } \\
\hline & $1-20$ & $1-20$ & $21-41$ & $42-61$ & $62-81$ \\
Rang & $21-41$ & 6 & 4 & 8 & 2 \\
1997-2001 & $42-61$ & 4 & 7 & 5 & 5 \\
& $62-81$ & 4 & 7 & 4 & 8 \\
\hline
\end{tabular}


Analog zu CARHART (1997) werden die Verwaltungsräte in Quartile eingeteilt, um die Abhängigkeit zwischen der ersten und der zweiten Subperiode zu untersuchen. Tabelle 4 zeigt, dass sechs der besten zwanzig Verwaltungsräte der ersten Subperiode auch in der zweiten Subperiode unter den besten zu finden sind, während fünf Personen in beiden Subperioden unter den schlechtesten zwanzig waren. Falls eine Persistenz in der Performance besteht, müssten die einzelnen Verwaltungsräte in der Diagonale von oben links nach unten rechts gehäuft auftreten. Rein optisch fällt keine besonders ausgeprägte diagonale Häufung auf. Ein $\chi^{2}$-Test nach KANJI (1999) mit neun Freiheitsgraden führt zu einem Wert von 9.64, so dass die Nullhypothese von Unabhängigkeit in der Performance nicht verworfen werden kann. Da die Anzahl Elemente in der Matrix jedoch gering ist, muss dieses Resultat vorsichtig interpretiert werden. Zusätzlich wurde eine Unterteilung in zwei Quantile vorgenommen. Der $\chi^{2}$-Test führt zu einem Wert von 0.99; die Unabhängigkeit in der Performance kann auch in diesem Fall nicht verworfen werden.

Mit den durchgeführten Untersuchungen kann keine Persistenz der Performance festgestellt werden. Mit anderen Worten kann nicht davon ausgegangen werden, dass Verwaltungsräte, welche eine überdurchschnittliche Performance in einer Untersuchungsperiode aufweisen, auch in der nächsten Untersuchungsperiode überdurchschnittlich abschneiden werden. Dafür gibt es mehrere mögliche Erklärungen: Erstens ist es denkbar, dass der einzelne Verwaltungsrat nur beschränkten Einfluss auf die Unternehmung besitzt. Zweitens ist davon auszugehen, dass der Markt die Qualität des Verwaltungsratsgremiums in die Bewertung der Aktie der Unternehmung einbezieht. Um nach einer Periode der Überperformance wieder eine Überperformance zu erzielen, müsste also der Verwaltungsrat nicht nur eine überdurchschnittliche Performance an den Tag legen, sondern auch die Erwartungen des Marktes an seine Performance übertreffen. Drittens ist es plausibel, dass die Performance des einzelnen Verwaltungsrates selbst Schwankungen über die Zeit unterworfen ist. Ähnlich wie ein Portfoliomanager, dessen Selektionserfolg über die Zeit schwankt, unterliegt auch die Qualität von Verwaltungsratsentscheiden gewissen Schwankungen.

\section{Personenübergreifende Performanceanalyse}

In diesem Abschnitt soll die Frage untersucht werden, ob besonders aktive Verwaltungsräte performancemässig bessere Resultate erzielen als solche, die sich auf wenige Mandate beschränken. Die Überprüfung dieser Fragestellung soll einerseits mit den Daten pro untersuchte Person als Grundgesamtheit und andererseits als Querschnittsvergleich aller jährlichen Beobachtungen pro Person durchgeführt werden.

\subsection{Querschnittsuntersuchung der Verwaltungsräte}

Die Aktivität eines Verwaltungsrates wird als Anzahl Mandate, die er bei verschiedenen Unternehmen ausübt, definiert. Sie wird mit den zwei Indikatoren „durchschnittliche Anzahl Mandatsjahre“ und „Anzahl verschiedene Unternehmen“ gemessen. Die durchschnittliche Anzahl Mandatsjahre bezeichnet den Mittelwert der geleisteten Mandatsjahre in der Beobachtungsperiode (eine einjährige Tätigkeit in einem Verwaltungsrat entspricht einem Mandatsjahr; eine einjährige gleichzeitige Tätigkeit in zwei Verwaltungsräten zwei Mandatsjahren, etc.). Unter der Anzahl verschiedener Unternehmen wird die Summe der verschiedenen Gesellschaften verstanden, bei denen eine Person im Beobachtungszeitraum als Verwaltungsrat tätig war. 
Performance wird wiederum als Aktienrendite definiert. Aufgrund der höheren Aussagekraft in Bezug auf die Leistung des einzelnen Verwaltungsratmitgliedes werden für die Überprüfung der Nullhypothese, dass zwischen Aktivität und Performance keine Abhängigkeit besteht, die Durchschnittsrenditen relativ zum SPI verwendet.

Eine Gruppierung der Stichprobe anhand der durchschnittlichen jährlichen Anzahl Mandatsjahre in Tabelle 5 zeigt, dass sich die Renditen, welche in den vier Gruppen erzielt wurden, statistisch nicht signifikant von der durchschnittlichen Rendite von $-5.81 \%$ unterscheiden. Zwischen der durchschnittlichen Aktivität von Verwaltungsräten und der durchschnittlichen Performance kann auf diese Weise kein Zusammenhang nachgewiesen werden. Die beiden Gruppen mit der grösseren Anzahl Mandatsjahre weisen allerdings unterdurchschnittliche Überrenditen auf, während die Gruppen mit wenig Mandatsjahren überdurchschnittliche Überrenditen erzielen.

Tabelle 5: Renditevergleich unterschiedlich aktiver Verwaltungsräte

\begin{tabular}{lccc}
\hline $\begin{array}{c}\text { Durchschnittliche Anzahl } \\
\text { Mandatsjahre }\end{array}$ & Anzahl Personen & $\begin{array}{c}\text { Anzahl Beobachtungen } \\
\text { (Mandatsjahre) }\end{array}$ & Überrendite \\
\hline 5.5 bis 3.0 & 24 & 902 & -5.91 \\
& & & $(-0.07)$ \\
2.9 bis 1.8 & 24 & 558 & $-7.22 \%$ \\
& & & $(-0.87)$ \\
1.7 bis 1.1 & 25 & 357 & $-3.68 \%$ \\
1.0 bis 0.3 & 22 & 167 & $(1.05)$ \\
Total & 95 & 1984 & $(0.51)$ \\
Alle Grössen sind annualisiert & & & $-5.81 \%$ \\
Renditen relativ zum SPI & & & \\
Beobachtungszeitraum 1.1.92 bis 31.12.01 & \\
t-Statistiken in Klammer unter den geschätzten Koeffizienten & & \\
\hline
\end{tabular}

Der Zusammenhang zwischen Aktivität und Rendite wird anhand zweier linearen Einfachregressionen mit der durchschnittlichen Anzahl Mandatsjahre, respektive der Anzahl verschiedener Unternehmen, als unabhängige Variable und der Performance je Verwaltungsrat als abhängige Variable untersucht:

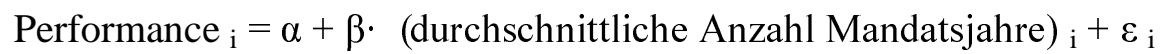

Performance $_{\mathrm{i}}=\alpha+\beta$. (Anzahl verschiedener Unternehmen $)_{\mathrm{i}}+\varepsilon_{\mathrm{i}}$

wobei: $\quad$ Performance $_{\mathrm{i}}$ : Überrendite des Veraltungsrates i relativ zum SPI

$\alpha$ : Achsenabschnitt

$\beta$ : Steigungskoeffizient

$\varepsilon_{\mathrm{i}}:$ Residuum

Die Resultate in Tabelle 6 zeigen einen schwach negativen Zusammenhang zwischen Aktivität und Performance. Die durchschnittliche Anzahl Mandatsjahre ist statistisch nicht signifikant, während die Anzahl Mandate bei verschiedenen Unternehmen immerhin eine Signifikanz auf dem Niveau von 10\% aufweist. Dies deutet auf einen ungünstigen Einfluss von vermehrter Mandatstätigkeit auf die Performance hin. 
Tabelle 6: Regressionsanalyse zwischen Performance und Aktivität der Verwaltungsräte

\begin{tabular}{lcc}
\hline & $\begin{array}{c}\text { Regression der Performance auf } \\
\text { die durchschnittliche Anzahl Man- } \\
\text { datsjahre }\end{array}$ & $\begin{array}{c}\text { Regression der Performance auf } \\
\text { die Anzahl Mandate bei verschie- } \\
\text { denen Unternehmen }\end{array}$ \\
\hline A & -0.0270 & -0.0150 \\
& $(-1.36)$ & $(-0.65)$ \\
Durchschnittliche Anzahl Mandatsjahre $(\beta)$ & -0.0110 & - \\
& $(-1.33)$ & $-0.0081^{*}$ \\
Mandate bei verschiedenen Unternehmen $(\beta)$ & - & $(-1.65)$ \\
Bestimmtheitsmass $\mathrm{R}^{2}$ & 0.019 & 0.028 \\
Prüfgrösse F & 1.759 & 2.719 \\
Anzahl Beobachtungen & 95 & 95 \\
\hline Alle Grössen sind annualisiert & & \\
Renditen relativ zum SPI & & \\
Beobachtungszeitraum 1.1.92 bis 31.12.01 & & \\
t-Statistiken in Klammer unter den geschätzten Koeffizienten & & \\
* bedeutet Signifikanz auf dem Niveau von 10\% & & \\
\hline
\end{tabular}

\subsection{Querschnittsuntersuchung aller Beobachtungen}

Da die meisten Verwaltungsräte nicht über die gesamte Untersuchungsperiode gleich viele Mandate ausüben, besteht die Gefahr, dass bei obiger Berechnungsweise, aufgrund der Durchschnittsbetrachtung pro Verwaltungsrat, Daten komprimiert werden und somit nicht richtig interpretiert werden können. Um diese Problematik zu umgehen, werden für diese Analyse die Daten nicht mehr pro Person aggregiert, sondern jährlich, auf Basis der einzelnen Verwaltungsräte, in die Untersuchung einbezogen. Die Aktivität wird also in diesem Sinne mit der Anzahl der in einem Jahr gleichzeitig ausgeübten Mandate gemessen und die Performance mit dem entsprechenden Renditemittelwert. Die Unternehmensrenditen werden dabei erneut im Vergleich zum SPI berechnet. Durch dieses Vorgehen erhöht sich die Anzahl der Beobachtungen um den Faktor der betrachteten Jahre (für einen zehnjährigen Untersuchungszeitraum um das zehnfache). Die beobachteten Wertepaare werden wiederum mit einer linearen Einfachregression gemäss obigem Schema auf ihren Zusammenhang hin untersucht.

In Tabelle 7 ist ein Trend erkennbar, dass Verwaltungsräte in Jahren mit wenigen Mandaten tendenziell eine bessere Performance erzielen als in Jahren mit höherer Belastung. Vom Mittelwert von $-5.81 \%$ verschieden ist nur der Wert für genau ein Verwaltungsratsmandat auf einem Signifikanzniveau von 10\%. Es kann also zumindest festgestellt werde, dass in Jahren mit nur einem Mandat die Unterperformance relativ zum SPI signifikant geringer ist als in Perioden mit mehreren Mandaten.

Tabelle 7: Quervergleich der relativen Renditen bezüglich der jährlichen Mandatstätigkeit

\begin{tabular}{|c|c|c|c|c|}
\hline $\begin{array}{l}\text { Anzahl Mandate } \\
\text { gleichzeitig }\end{array}$ & $\begin{array}{l}\text { Anzahl Beob- } \\
\text { achtungen }\end{array}$ & $\begin{array}{l}\text { Anteil an Grundge- } \\
\text { samtheit }\end{array}$ & $\varnothing$-Rendite & t-Statistiken \\
\hline 8 & 1 & $0.1 \%$ & $-1.65 \%$ & 0.31 \\
\hline 7 & 4 & $0.4 \%$ & $-13.94 \%$ & -1.12 \\
\hline 6 & 27 & $2.8 \%$ & $-4.27 \%$ & 0.51 \\
\hline 5 & 57 & $6.0 \%$ & $-9.21 \%$ & -1.50 \\
\hline 4 & 94 & $9.9 \%$ & $-7.95 \%$ & -1.09 \\
\hline 3 & 156 & $16.4 \%$ & $-6.20 \%$ & -0.22 \\
\hline 2 & 217 & $22.8 \%$ & $-3.73 \%$ & 1.13 \\
\hline 1 & 223 & $23.5 \%$ & $-1.33 \%{ }^{*}$ & 1.75 \\
\hline 0 & 171 & $18.0 \%$ & - & - \\
\hline Total & 950 & $100.0 \%$ & $-5.81 \%$ & \\
\hline
\end{tabular}

Alle Grössen sind annualisiert

Renditen relativ zum SPI

Beobachtungszeitraum 1.1.92 bis 31.12 .01

* bedeutet Signifikanz auf dem Niveau von $10 \%$ 
Eine entsprechende Regression mit der Anzahl gleichzeitig ausgeübter Mandate als unabhängige Variable und der durchschnittlichen relativen Rendite als abhängige Variable bestätigt dieses Resultat: Zwischen der Anzahl gleichzeitig ausgeübter Mandate und der Performance (gemessen als relative Rendite) besteht ein statistisch signifikanter, negativer Zusammenhang. Die Regressionsparameter sind in Tabelle 8 ersichtlich.

Tabelle 8: Regressionsanalyse zwischen Performance und Aktivität auf jährlicher Basis relativ SPI

\begin{tabular}{|c|c|}
\hline & $\begin{array}{l}\text { Regression der Performance auf die Anzahl } \\
\text { gleichzeitig ausgeübte Mandate }\end{array}$ \\
\hline$\alpha$ & $\begin{array}{c}-0.004 \\
(-0.244)\end{array}$ \\
\hline Anzahl gleichzeitig ausgeübte Mandate $(\beta)$ & $\begin{array}{l}-0.016^{* \star *} \\
(-2.623)\end{array}$ \\
\hline Bestimmtheitsmass $\mathrm{R}^{2}$ & 0.009 \\
\hline Prüfgrösse $F$ & 6.87 \\
\hline
\end{tabular}

Somit muss die Nullhypothese, dass zwischen der Aktivität und der Performance kein Zusammenhang besteht, verworfen werden: Die Anzahl gleichzeitig ausgeübter Mandate beeinflusst die Performance statistisch signifikant negativ. Personen, die in mehreren Verwaltungsratsgremien von kotierten Schweizer Gesellschaften gleichzeitig vertreten sind, erzielen schlechtere Resultate als solche, die sich nur auf wenige Mandate gleichzeitig konzentrierten. Der Steigungskoeffizient zeigt, dass jedes zusätzliche Verwaltungsratsmandat die jährliche Überperformance zum SPI um 1.6\% schmälert. Die Regression liefert allerdings nur einen sehr geringen Erklärungsbeitrag (Bestimmtheitsmass <1\%) für die unterschiedlichen Renditen der insgesamt 779 Beobachtungen (95 Verwaltungsräte à 10 Jahre abzüglich Beobachtungen ohne Mandate) und muss deshalb vorsichtig interpretiert werden. Die Erkenntnis ist dennoch sehr aufschlussreich, da ein solcher Zusammenhang bisher empirisch nicht nachgewiesen werden konnte.

Diese Beobachtung steht im Einklang mit der These, welche der Unabhängigkeit von Verwaltungsratsmitgliedern grosse Bedeutung in Bezug auf eine effektive Corporate Governance und somit indirekt auch auf die Unternehmensperformance beimisst. Wenn ein Aktionärsvertreter mehrere Mandate gleichzeitig ausübt, erhöht sich die Gefahr von potentiellen Interessenkonflikten und Abhängigkeiten. Eine weitere Begründung für diesen Zusammenhang könnten auch Zeitaspekte sein, da Verwaltungsratsmandate normalerweise nur im Nebenamt ausgeführt werden. Ein Verwaltungsrat, der neben seiner Haupttätigkeit noch bei drei oder vier kotierten Gesellschaften im Aufsichtsorgan Einsitz nimmt, kann sich weit weniger intensiv mit dem jeweiligen Unternehmen befassen als er dies mit weniger Mandaten könnte. Die vorliegende Studie kommt somit zu einem anderen Resultat als die ansatzweise ähnliche empirische Untersuchung von DOWEN (1995).

\section{Schlusswort und Ausblick}

Im Rahmen einer Stichprobe von 95 Verwaltungsratsmitgliedern von börsenkotierten Schweizer Firmen wurde für den Zeitraum von Anfang 1992 bis Ende 2001 untersucht, ob es Personen gibt, die mit Verwaltungsratsmandaten eine überdurchschnittliche Performance erzielt haben. Dazu wurde die Performance einerseits anhand der wechselnden Zusammensetzung der jeweiligen Mandatsportfolios im Verlaufe des zehnjährigen Beobachtungszeitraumes und andererseits als Durchschnitt der ausgeübten Mandate berechnet. 
Zum Teil gibt es beträchtliche Performanceunterschiede innerhalb der Stichprobe, und auch die verschiedenen Berechnungsverfahren führen zu teilweise unterschiedlichen Resultaten für die einzelnen Personen. Einige Verwaltungsräte haben sowohl relativ zum Stichprobenmittelwert als auch im Vergleich zu einem Benchmark bedeutend schlechter abgeschnitten. Anhand der Analyse der Performancenachhaltigkeit, hat sich gezeigt, dass zwischen historischer und zukünftiger Performance kein Zusammenhang festgestellt werden kann. Eine gute Performance in einer Periode ist offenbar kein Indikator für eine gute Performance im folgenden Zeitintervall. Diese Erkenntnisse zeigen, dass die langfristige Unternehmensperformance zwar zum Teil von den jeweiligen Verwaltungsratsmitgliedern abhängig ist; sie kann jedoch nur in einem beschränkten Ausmass von ihnen beeinflusst werden. Systematisch überdurchschnittliche Fähigkeiten konnten mit den verwendeten Methoden nicht festgestellt werden.

$\mathrm{Zu}$ einem interessanten Ergebnis kommt die personenübergreifende Analyse der erzielten Performance: Zwischen der Anzahl gleichzeitig ausgeübter Mandate und der Performance kann empirisch ein statistisch signifikanter, negativer Zusammenhang nachgewiesen werden. Das heisst, der Performancedurchschnitt ist umso schlechter, je mehr Mandate eine Person gleichzeitig ausführt. Zwar liefert dieser Zusammenhang nur einen sehr kleinen Erklärungsbeitrag der Performancestreuung, widerlegt jedoch die Hypothese, wonach besonders aktive Verwaltungsräte aufgrund ihrer Erfahrung und ihrem grossen Netzwerk besonders erfolgreich sind. Dieser empirische Befund impliziert, dass unter Umständen bei der Wahl von Verwaltungsratsmitgliedern der Unabhängigkeit und zeitlichen Verfügbarkeit mehr Bedeutung als der Popularität und dem mit der Person verknüpften Zugang zu Ressourcen beigemessen werden sollte.

Umfassendere Ergebnisse sind durch eine Ausweitung der Untersuchung auf sämtliche Verwaltungsräte von SMI Firmen, auch solche mit nur einem Mandat, zu erwarten. Auch könnte die für die Performance massgebliche Zeitperiode auf den Zeitpunkt des Eintritts, der Wahl oder der Ankündigung des Eintritts eines neuen Verwaltungsrates ausgedehnt werden. Die Berücksichtigung verschiedener Funktionen von Verwaltungsräten wie Präsident oder Mitglied eines Ausschusses, deren Eigenschaften (Verweildauer im Verwaltungsrat, Alter, Bildung etc.) sowie der Anzahl Verwaltungsräte einer Firma könnte ebenfalls neue Erkenntnisse bringen.

\section{FUSSNOTEN}

[1] Ausnahmen bestehen für Investmentgesellschaften und seit Oktober 2001 für Titel mit einem zu geringen Free-Float.

[2] Die absolute Rendite der Verwaltungsratsportfolios wurde ebenfalls berechnet, mit unterschiedlichen Resultaten. Da aber die Verwaltungsräte in verschiedenen Marktphasen aktiv waren, sind die Resultate nur beschränkt aussagekräftig und werden deshalb nicht dargestellt.

[3] Eine Performancemessung relativ zur Branche führt zu stark abweichenden Resultaten im Vergleich zur Analyse der Überrendite zum SPI. Aus den genannten Gründen wurde sie hier nicht publiziert.

\section{QUELLEN}

BALIGA, R., C. MOYER und R. RAO (1996): „CEO Duality and Firm Performance: What's the Fuss?“, Strategic Management Journal, S. 41-53. 
BAYSINGER, R. und H. BUTLER (1985): „Corporate Governance and the Board of Directors: Performance Effects of Changes in Board Composition“, Journal of Law, Economics and Organization, S. 101-124.

BRICKLEY, J., J. COLES und G. JARREL (1997): „Leadership Structure: Separating the CEO and Chairman of the Board“, Journal of Corporate Finance, S. 189-220.

BURT, R. (1983): „Corporate Profits and Cooptation: Network of Market Constraint and Directorate Ties in the American Economy“, New York: Academic Press.

CARRINGTON, P. (1981): „Horizontal Co-optation through Corporate Interlocks“, Dissertation, University of Toronto.

DAHYA, J. und N. TRAVLOS (2000): „Does the One Man Show Pay? Theory and Evidence on the Dual CEO Revisited“, European Financial Management, S. 85-98.

DEMB, A. und F. NEUBAUER (1992): „The Corporate Board: Confronting the Paradoxes“, Long Range Planning, S. 9-20.

DONALDSON, L. (1990): „The Ethereal Hand: Organizational Economics and Management Theory“, Academy of Management Review, S. 369-381.

DOWEN, R. (1995): „Board of Director Quality and Firm Performance“, International Journal of the Economics of Business, S. 123-132.

DRUMMEN, M. und H. ZIMMERMANN (1992): „The Structure of European Stock Returns“, Financial Analysts Journal, July-August, S. 15-26.

EISENBERG, T., S. SUNDGREN und M. WELLS (1998): „Larger Board Size and Decreasing Firm Value in Small Firms“, Journal of Financial Economics, S. 35-54.

FLIGSTEIN, N. und P. BRANTLEY (1992): „Bank Control, Owner Control or Organizational Dynamics: Who Controls the Large Modern Corporation?", American Journal of Sociology, S. 280-307.

GRÜNBICHLER, A. und P. OERTMANN (1995): „Corporate Governance: Schweizer Erfahrungen“ Working Paper, Universität St. Gallen.

HERMALIN, B. und M.WEISBACH (1991): ,The Effects of Board Composition and Direct Incentives on Firm Performance" “ Financial Management, S. 101-112.

JENSEN, M. (1968): „The Performance of Mutual Funds in the Period 1945-1964“, Journal of Finance, S. 389-416.

JENSEN, M. (1993): „The Modern Industrial Revolution and Failure of Internal Control Systems", Journal of Finance, S. 389-416.

JOBSON, J. und B. KORKIE (1981): „Performance Hypothesis Testing with the Sharpe and Treynor Measures“, Journal of Finance, S. 889-908.

JOHNSON, J., C. DAILY und A. ELLSTRAND (1996): „Boards of Directors: A Review and Reasearch Agenda“, Journal of Management, S. 409-438.

JUDGE, W. und C. ZEITHAML (1992): „Institutional and Strategic Choice Perspectives on Board Involvement in the Strategic Decision Process“, Academy of Management Journal, S. 766794.

KANJI, G.K. (1999): „100 Statistical Tests“, London: SAGE.

LORSCH, J. und E. MACIVER (1989): „Pawns of Potentates: The Reality of America's Corporate Boards“, Harvard Business School Press, Boston.

MACE, M. (1971): „Directors: Myth and Reality“, Boston: Harvard Business School Press.

MIZRUCHI, M. (1996): „What do Interlocks do? An Analysis, Critique, and Assessment of Research on Interlocking Directorates“, Annual Review of Sociology, S. 271-298.

o. V. (o. J.): ,Schweizerisches Obligationenrecht“, SR 220.

o. V. (1991-2000): „Verzeichnis der Verwaltungsräte“, Zürich: Orell Füssli Verlag. 
o. V. (2002): "Rating der Verwaltungsräte 2002", Leadership, Knight Gianella \& Partner AG, S. 6-9.

o. V. (2002): „SPI Titel per Ende 2001“, Schweizer Börse, www.swx.com.

PALMON, O. und J. WALD (2002): „Are Two Heads Better than One? The Impact of Changes in Management Structure on Performancer by Firm Size“, Journal of Coporate Finance, S. 213226.

PENNINGS, J. (1980): „Interlocking Directorates“, San Francisco: Jossey-Bass.

PFANNSCHMIDT, A. (1993): „Personelle Verflechtungen über Aufsichtsräte“, Wiesbaden:

Gabler.

PFEFFER, J. und G. SALANCIK (1978): „The External Control of Organizations - A Resource Dependence Perspective“, New York: Harper \& Row.

RECHNER, P. und D. DALTON (1991): „CEO Duality and Organizational Performance: A Longitudinal Analysis“, Strategic Management Journal, S. 155-160.

ROSENSTEIN, J., A.V. BRUNO, W.D. BYGRAVE und N.T. TAYLOR (1993): „The CEO, Venture Capitalists, and the Board“" Journal of Business Venturing, S. 99-113.

ROSENSTEIN, S.und J. WYATT (2001): „Outside Directors, Board Independence and Shareholder Wealth“, Journal of Fiancial Economics, S. 175-191.

SCHAFFER, B. (2002): „Board Assessments of Managerial Performance“, Journal of Managerial Psychology, S. 95-115.

SCHWARZ, G. (2001): „Filz, Kontrolle und Verantwortung“, Neue Zürcher Zeitung, 24. 3. 2001, S. 21.

SHARPE, W. (1966): „Mutual Fund Performace“, Journal of Business, S. 119-138.

TREYNOR, J. und F. BLACK (1973): "How to Use Security Analysis to Improve Portfolio Selsection“, Journal of Business, S. 66-86.

WEIBEL, P. (1998): „Strategieentwicklung und -durchsetzung“, in: S. Biland und M. Hilber (Hrsg.): Verwaltungsrat als Gestaltungsrat, Zürich: WEKA Verlag, S. 103-112.

YERMACK, D. (1996): „Higher Market Valuation of Companies with a Small Board of Directors“, Journal of Financial Economics, S. 185-211.

ZAJAC, E. (1988): „Interlocking Directorates as an Interorganizational Strategy: A Test of Critical Assumptions“, Academy of Management Journal, S. 428-238. 
ANHANG

Übersicht der Verwaltungsräte mit den entsprechenden Mandaten und Zeiträumen.

\begin{tabular}{|c|c|c|c|}
\hline Name & Vorname & Gesellschaft & Amtszeit \\
\hline \multirow[t]{5}{*}{ Ackermann } & Josef & ABB & 1994-1996 \\
\hline & & CKW & 1995-1997 \\
\hline & & Elektrowatt & $1996 / 1997$ \\
\hline & & Swissair & $1996-2000$ \\
\hline & & Unaxis (ehemals Oerlikon-Bührle) & 1992-1995 \\
\hline \multirow[t]{2}{*}{ Albers } & Ulrich & ABB & 1992 \\
\hline & & Credit Suisse Group & 1992-1999 \\
\hline \multirow[t]{5}{*}{ Amstutz } & Marc & Alusuisse & $1992-2000$ \\
\hline & & $\mathrm{HCB}$ & 1992-1994 \\
\hline & & Holcim & $1992-2000$ \\
\hline & & SGS & $2000 / 2001$ \\
\hline & & Von Roll & $1994-2000$ \\
\hline \multirow[t]{2}{*}{ Angst } & Carl & SBV & 1992 \\
\hline & & Sulzer & 1992-1995 \\
\hline \multirow[t]{7}{*}{ Bechtler } & Thomas & Bank Leu & $1992 / 1993$ \\
\hline & & Bucher & 1992-2001 \\
\hline & & Conzetta & $1992-2001$ \\
\hline & & Credit Suisse Group & $1995-2001$ \\
\hline & & Sika & 1992-2001 \\
\hline & & Swiss $\operatorname{Re}$ & $1995-2001$ \\
\hline & & Zellweger & $1992-2001$ \\
\hline \multirow[t]{4}{*}{ Binkert } & Pius & Alusuisse & 1992 \\
\hline & & Forbo & 1992-1994 \\
\hline & & Keramik Laufen & 1992-1998 \\
\hline & & SBV & 1992-1994 \\
\hline \multirow[t]{8}{*}{ Blocher } & Christoph & Alusuisse & 2000 \\
\hline & & Arbonia-Forster & 1992-1997 \\
\hline & & Ems-Chemie & $1992-2001$ \\
\hline & & Lonza & $2000 / 2001$ \\
\hline & & Motor-Columbus & 1992 \\
\hline & & Netstal & 1994-1999 \\
\hline & & Pharma Vision & $1992-1999$ \\
\hline & & SBG & 1992 \\
\hline \multirow[t]{8}{*}{ Blum } & Georges & ABB & $1995 / 1996$ \\
\hline & & Agefi & 2001 \\
\hline & & Energie Simplon & $1992-2001$ \\
\hline & & Nestlé & $1998 / 1999$ \\
\hline & & SBV & 1997 \\
\hline & & Sulzer & $1995-2001$ \\
\hline & & Swissair & $1995-2000$ \\
\hline & & Unigestion & 1992-1994, 2000 \\
\hline \multirow[t]{5}{*}{ Böckli } & Peter & Nestlé & 1994-2001 \\
\hline & & Sandoz & 1992-1994 \\
\hline & & SBV & $1992-1997$ \\
\hline & & Suter \& Suter & 1992-1994 \\
\hline & & UBS & $1999-2001$ \\
\hline
\end{tabular}




\begin{tabular}{|c|c|c|c|}
\hline & & Zürich Versicherungen & $1992-2000$ \\
\hline \multirow[t]{4}{*}{ Bodmer } & Albert & Alusuisse & $1992-1997$ \\
\hline & & Ciba-Geigy & 1992 \\
\hline & & Credit Suisse Group & 1992 \\
\hline & & Helvetia & 1992 \\
\hline \multirow[t]{4}{*}{ Bodmer } & Henry & Credit Suisse Group & $1992-1996$ \\
\hline & & Huber \& Suhner & $1992-1995$ \\
\hline & & Pirelli & 1992 \\
\hline & & Zürich Versicherungen & 1992-2001 \\
\hline \multirow[t]{6}{*}{ Borgeaud } & Pierre & Centerpulse (ehemals Sulzer Medica) & $1998-2001$ \\
\hline & & Clariant & $1996-2001$ \\
\hline & & Pirelli & $1992-1998$ \\
\hline & & SBV & 1992-1997 \\
\hline & & Sulzer & $1992-2001$ \\
\hline & & Winterthur & $1992-1996$ \\
\hline \multirow[t]{3}{*}{ Brabeck } & Peter & Credit Suisse Group & $1998-2001$ \\
\hline & & Nestlé & $1999-2001$ \\
\hline & & Roche & 2001 \\
\hline \multirow[t]{7}{*}{ Bremi } & Ulrich & Conzetta & 1992 \\
\hline & & Credit Suisse Group & $1992-2000$ \\
\hline & & Elektrowatt & 1992 \\
\hline & & Georg Fischer & 1992-1998 \\
\hline & & Kaba & $1997-2001$ \\
\hline & & NZZ & 1995 \\
\hline & & Swiss $R e$ & $1994-2000$ \\
\hline \multirow[t]{4}{*}{ Bühler } & Urs & Clariant & $1996 / 1997$ \\
\hline & & SBV & $1992-1996$ \\
\hline & & Sulzer & $1992-2000$ \\
\hline & & Winterthur & $1992-1996$ \\
\hline \multirow[t]{5}{*}{ Cappis } & Marc & Bobst & $1997-2001$ \\
\hline & & Huber \& Suhner & $1992-2001$ \\
\hline & & Mikron & $1992-1996$ \\
\hline & & SBG & $1992-1997$ \\
\hline & & Swissair & $1992-2000$ \\
\hline \multirow[t]{4}{*}{ Cassani } & Kaspar & Ciba-Geigy & $1992-1995$ \\
\hline & & Credit Suisse Group & $1992-1996$ \\
\hline & & Novartis & 1997-1999 \\
\hline & & Zürich Versicherungen & $1994-2000$ \\
\hline \multirow[t]{7}{*}{ Chaudet } & Marc-Henri & Credit Suisse Group & $1999-2001$ \\
\hline & & Orell Füssli & $1992-2000$ \\
\hline & & Orior & 1994-1999 \\
\hline & & Pargesa & $1998-2001$ \\
\hline & & Romande Energie & 1998 \\
\hline & & Vaudoise d'Electricité & $1999-2001$ \\
\hline & & Winterthur & $1992-1996$ \\
\hline \multirow[t]{3}{*}{ de Kalbermatten } & Bruno & Bobst & $1992-2000$ \\
\hline & & Nestlé & 1992-1997 \\
\hline & & SBG & 1992-1994 \\
\hline \multirow[t]{3}{*}{ de Pury } & David & ABB & $1992-1996$ \\
\hline & & Ciba-Geigy & $1994 / 1995$ \\
\hline & & Credit Suisse Group & 1994-1996 \\
\hline
\end{tabular}




\begin{tabular}{|c|c|c|c|}
\hline & & Nestlé & $1994-2001$ \\
\hline & & Zürich Versicherungen & 1994-2001 \\
\hline \multirow[t]{2}{*}{ Diehl } & Walter & Credit Suisse Group & $1992-1994$ \\
\hline & & Swiss Re & 1992 \\
\hline \multirow[t]{8}{*}{ Doerig } & Hans-Ulrich & Alusuisse & $1992-2000$ \\
\hline & & Clariant & 1996/1997 \\
\hline & & EG Laufenburg & $1994-1998$ \\
\hline & & Elektrowatt & $1992-1997$ \\
\hline & & Konsumverein & 1994 \\
\hline & & KW Laufenburg & $1992-1998$ \\
\hline & & Novartis & $1998-2001$ \\
\hline & & Orell Füssli & 1992 \\
\hline \multirow[t]{5}{*}{ Domeniconi } & Reto & Bobst & $1995-2001$ \\
\hline & & Centerpulse & $1998-2001$ \\
\hline & & Nestlé & $1998-2001$ \\
\hline & & Sulzer & 1996-2001 \\
\hline & & Xstrata & $1998-2001$ \\
\hline \multirow[t]{2}{*}{ Dunkel } & Arthur & Credit Suisse Group & $1995-2001$ \\
\hline & & Nestlé & $1995-2001$ \\
\hline \multirow[t]{8}{*}{ Ebner } & Martin & ABB & $2000 / 2001$ \\
\hline & & Alusuisse & 2000 \\
\hline & & BK Vision & 1994-2001 \\
\hline & & Lonza & $2000 / 2001$ \\
\hline & & OZ Holding & $1992-1999$ \\
\hline & & Pharmavision & $1994-2001$ \\
\hline & & Spezialitäten Vision & 1995-2001 \\
\hline & & Stillhalter Vision & 1995-2001 \\
\hline \multirow[t]{6}{*}{ Feller } & Kurt & Ciba SC & 2001 \\
\hline & & Dätwyler & 1992-2001 \\
\hline & & Geberit & 2001 \\
\hline & & Rieter & 1995-2001 \\
\hline & & SBG & 1992-1997 \\
\hline & & Scintilla & $2000 / 2001$ \\
\hline \multirow[t]{7}{*}{ Frehner } & Walter & Baloise & 1992-1996, 1999-2001 \\
\hline & & Ciba-Geigy & 1995 \\
\hline & & Nestlé & 1994-1997 \\
\hline & & Novartis & $1997-2001$ \\
\hline & & SBV & 1994-1996 \\
\hline & & Schindler & $1992-2001$ \\
\hline & & Swatch & $1992-1997$ \\
\hline \multirow[t]{6}{*}{ Galliker } & Franz & ABB & 1992-1994 \\
\hline & & Alusuisse & 1992-1995 \\
\hline & & Ciba-Geigy & $1992-1994$ \\
\hline & & Holvis & 1992-1994 \\
\hline & & SBV & 1992-1994 \\
\hline & & Sulzer & $1992-1994$ \\
\hline \multirow[t]{2}{*}{ Gasser } & Rupert & Alusuisse & 1994-2000 \\
\hline & & Lonza & $2000 / 2001$ \\
\hline \multirow[t]{3}{*}{ Genillard } & Robert & Bank Leu & $1992 / 1993$ \\
\hline & & Credit Suisse Group & $1992-2000$ \\
\hline & & Novartis & $1998-2001$ \\
\hline
\end{tabular}


Sandoz

1992-1994

\begin{tabular}{|c|c|c|c|}
\hline \multirow{6}{*}{ Gerber } & & & \\
\hline & Fritz & Credit Suisse Group & 1992 \\
\hline & & Nestlé & $1995-2001$ \\
\hline & & Roche & $1992-2001$ \\
\hline & & Victoria-Jungfrau & 1994-1997 \\
\hline & & Zürich Versicherungen & $1992-1996$ \\
\hline \multirow[t]{6}{*}{ Gisling } & Jean-Claude & Clariant & $1996-1999$ \\
\hline & & Credit Suisse Group & 1992-1994 \\
\hline & & Publigroupe & $1992-1994$ \\
\hline & & Swissair & $1992-1995$ \\
\hline & & Vetropack & $1992-2001$ \\
\hline & & Von Roll & $1992-1995$ \\
\hline \multirow[t]{7}{*}{ Goetz } & Hannes & Georg Fischer & $1992,1997-2001$ \\
\hline & & Montreux Palace & $1995-2000$ \\
\hline & & NZZ & 1995 \\
\hline & & SBG & $1992-1997$ \\
\hline & & SIG & $1992-2001$ \\
\hline & & Suter \& Suter & $1992-1995$ \\
\hline & & Swissair & $1992-2000$ \\
\hline \multirow[t]{2}{*}{ Grete } & Ulrich & Alusuisse & $1994-1998$ \\
\hline & & SGS & 1992-1996, 2000 \\
\hline \multirow[t]{2}{*}{ Grether } & Esther & SBV & $1992-1996$ \\
\hline & & Swatch & $1992-2001$ \\
\hline \multirow[t]{8}{*}{ Gugler } & Adolf & Bank Leu & $1992 / 1993$ \\
\hline & & CKW & $1992-2000$ \\
\hline & & Credit Suisse Group & $1995-2000$ \\
\hline & & Dätwyler & $1994-2000$ \\
\hline & & EG Laufenburg & $1992-1994$ \\
\hline & & Elektrowatt & $1992-1997$ \\
\hline & & KW Laufenburg & $1992-1996$ \\
\hline & & Unaxis & $1992-1999$ \\
\hline \multirow[t]{6}{*}{ Gut } & Rainer & Ciba-Geigy & $1992-1995$ \\
\hline & & Credit Suisse Group & $1992-2000$ \\
\hline & & Elektrowatt & $1992-1994$ \\
\hline & & Nestlé & $1994-2001$ \\
\hline & & Swiss Re & $1992-1997$ \\
\hline & & Swissair & $1992-1995$ \\
\hline \multirow[t]{3}{*}{ Haerri } & Herrmann & Alusuisse & $1992-1994$ \\
\hline & & Credit Suisse Group & 1992 \\
\hline & & Walter Rentsch Holding & $1992-1995$ \\
\hline \multirow[t]{3}{*}{ Hänggi } & Rolf & Eichhof & $1999-2001$ \\
\hline & & Roche & $1998-2001$ \\
\hline & & Zürich Versicherungen & $1995-1998$ \\
\hline \multirow[t]{3}{*}{ Hentsch } & Bendedict & Energie Simplon & $1994-2000$ \\
\hline & & Swiss Re & $1995-2001$ \\
\hline & & Swissair & $1992-2001$ \\
\hline \multirow[t]{2}{*}{ Hoffmann } & André & Givaudan & 2001 \\
\hline & & Roche & $1998-2001$ \\
\hline \multirow[t]{3}{*}{ Höhler } & Gertrud & Baloise & $2000 / 2001$ \\
\hline & & Ciba SC & 1998-2001 \\
\hline & & Georg Fischer & $2000 / 2001$ \\
\hline
\end{tabular}




\begin{tabular}{|c|c|c|c|}
\hline \multirow[t]{3}{*}{ Honegger } & \multirow[t]{3}{*}{ Eric } & Affichage & 2001 \\
\hline & & Swissair & $1995-2001$ \\
\hline & & UBS & $2000 / 2001$ \\
\hline \multirow[t]{2}{*}{ Humer } & \multirow[t]{2}{*}{ Franz } & Roche & $1997-2001$ \\
\hline & & Zürich Versicherungen & 2001 \\
\hline \multirow[t]{2}{*}{ Hüppi } & \multirow[t]{2}{*}{ Rolf } & SBV & $1995-1997$ \\
\hline & & Zürich Versicherungen & $1995-2001$ \\
\hline \multirow[t]{10}{*}{ Jeker } & \multirow[t]{10}{*}{ Robert } & $\mathrm{ABB}$ & $1992-2001$ \\
\hline & & Bank Leu & $1992 / 1993$ \\
\hline & & Batigroup & $1998-2001$ \\
\hline & & Elektrowatt & 1997 \\
\hline & & Georg Fischer & $1992-2001$ \\
\hline & & Landis \& Gyr & $1994 / 1995$ \\
\hline & & Pirelli & 1992 \\
\hline & & Real Estate Group & 2001 \\
\hline & & Stratec & $1997 / 1998$ \\
\hline & & Swiss Steel & 1998-2001 \\
\hline \multirow[t]{4}{*}{ Jetzer } & \multirow[t]{4}{*}{ Alexandre } & Bank Leu & $1992 / 1993$ \\
\hline & & Novartis & $1997-2001$ \\
\hline & & Sandoz & 1992-1995 \\
\hline & & Winterthur & $1992-1996$ \\
\hline \multirow[t]{2}{*}{ Jucker } & \multirow[t]{2}{*}{ Hans } & Alusuisse & $1992-1997$ \\
\hline & & SBG & $1992-1997$ \\
\hline \multirow[t]{3}{*}{ Junger } & \multirow[t]{3}{*}{ Martin } & Baloise & $1992-2001$ \\
\hline & & Crossair & 1992 \\
\hline & & SBG & 1992 \\
\hline \multirow[t]{2}{*}{ Kielholz } & \multirow[t]{2}{*}{ Walter } & Credit Suisse Group & 2001 \\
\hline & & Swiss $\operatorname{Re}$ & $2000 / 2001$ \\
\hline \multirow[t]{6}{*}{ Kissling } & \multirow[t]{6}{*}{ Willy } & Esec & 2001 \\
\hline & & Forbo & $1997-2001$ \\
\hline & & Holcim & $1998-2001$ \\
\hline & & Landis \& Gyr & $1992-1995$ \\
\hline & & Unaxis & $2000 / 2001$ \\
\hline & & Von Roll & $1998-2000$ \\
\hline \multirow[t]{5}{*}{ Krauer } & \multirow[t]{5}{*}{ Alex } & Baloise & $1992-2000$ \\
\hline & & Ciba Geigy & $1992-1995$ \\
\hline & & Novartis & $1997-2000$ \\
\hline & & SBV & $1992-1997$ \\
\hline & & UBS & 1999-2001 \\
\hline \multirow[t]{7}{*}{ Kündig } & \multirow[t]{7}{*}{ Markus } & Clariant & $1996-2001$ \\
\hline & & Landis \& Gyr & $1992-1995$ \\
\hline & & Metallwaren-Holding & $1992-2001$ \\
\hline & & Pelikan & $1992-2001$ \\
\hline & & SBG & $1992-1997$ \\
\hline & & UBS & $1999-2001$ \\
\hline & & Zürich Versicherungen & $1992-2000$ \\
\hline Küpfer & Peter & Bank Leu & 1992 \\
\hline & & Büro Fürrer & $1992-2001$ \\
\hline & & Elektrowatt & $1992-1997$ \\
\hline & & Esec & $2000 / 2001$ \\
\hline & & Julius Bär & 2001 \\
\hline
\end{tabular}




\begin{tabular}{|c|c|c|c|}
\hline & & Netstal & $2000 / 2001$ \\
\hline & & Swiss Steel & 1997 \\
\hline & & Swisscom & $2000 / 2001$ \\
\hline & & Unaxis & $2000 / 2001$ \\
\hline & & Valora & $2000 / 2001$ \\
\hline Landolt & Pierre & Novartis & $1997-2001$ \\
\hline & & Sandoz & 1992-1994 \\
\hline & & Syngenta & 2001 \\
\hline Languetin & Pierre & Pargesa & 1992-2001 \\
\hline & & Sandoz & $1992-1995$ \\
\hline & & Swiss Re & 1992-1994 \\
\hline Lehn & Jean-Marie & Ciba-Geigy & $1992-1995$ \\
\hline & & Ciba SC & $1998-2001$ \\
\hline Leuenberger & Andreas & Givaudan & 2001 \\
\hline & & Metallwaren-Holding & 2001 \\
\hline & & Rentenanstalt & 2001 \\
\hline & & Roche & $1992-2001$ \\
\hline & & Swissair & $1996-2001$ \\
\hline Leutwiler & Fritz & $\mathrm{ABB}$ & 1992 \\
\hline & & Ciba-Geigy & 1992-1994 \\
\hline & & Nestlé & $1992-1998$ \\
\hline & & Winterthur & $1992-1995$ \\
\hline Lippuner & Heini & Credit Suisse Group & $1994-2001$ \\
\hline & & Elektrowatt & 1992 \\
\hline & & Novartis & $1998-2001$ \\
\hline & & Winterthur & 1992-1996 \\
\hline Loepfe & Otto & Credit Suisse Group & 1992-1997 \\
\hline & & Crossair & 1995-1998 \\
\hline & & Swissair & $1997 / 1998$ \\
\hline & & Winterthur & 1992-1996 \\
\hline Marchionne & Sergio & Alusuisse & 2000 \\
\hline & & Lonza & $2000 / 2001$ \\
\hline & & Serono & 2001 \\
\hline Maucher & Helmut & ABB & 1994-1997 \\
\hline & & Credit Suisse Group & $1992-1997$ \\
\hline & & Montreux Palace & $1995-2000$ \\
\hline & & Nestlé & $1992-2000$ \\
\hline & & Zürich Versicherungen & 1992-1996 \\
\hline Meier & Henri & Givaudan & 2001 \\
\hline & & Roche & $1996-2001$ \\
\hline & & Victoria-Jungfrau & $1997-2001$ \\
\hline Meyer & Rolf Arthur & Ciba SC & $1998-2001$ \\
\hline & & SBG & 1994-1997 \\
\hline & & UBS & $1999-2001$ \\
\hline Milliet & François & Bobst & 1992-1997 \\
\hline & & Galenica & 1993-1996 \\
\hline & & Publigroupe & $1992-1996$ \\
\hline & & SBG & $1992-1995$ \\
\hline & & SGS & 1992-1996 \\
\hline Moret & Marc & Credit Suisse Group & 1992 \\
\hline & & Sandoz & 1992-1995 \\
\hline
\end{tabular}




\begin{tabular}{|c|c|c|c|}
\hline & & Swissair & $1992-1995$ \\
\hline \multirow[t]{3}{*}{ Mühlemann } & Lukas & Credit Suisse Group & 2001 \\
\hline & & Swiss $R e$ & $1996-2001$ \\
\hline & & Swissair & $1996-2001$ \\
\hline \multirow[t]{3}{*}{ Muller } & Georges & HPI Holding & $1995-1999$ \\
\hline & & Rentenanstalt & $1998-2001$ \\
\hline & & Serono & $1994-2001$ \\
\hline \multirow[t]{3}{*}{ Müller } & Erich & Credit Suisse Group & $1992-1996$ \\
\hline & & Mikron & $1992-2001$ \\
\hline & & Sulzer & $2000 / 2001$ \\
\hline \multirow[t]{2}{*}{ Müller-Berghof } & Bernd & ABB & $1992-1998$ \\
\hline & & SBG & 1992-1995 \\
\hline \multirow[t]{6}{*}{ Pozzi } & Angelo & Alusuisse & $1992-1994$ \\
\hline & & Atel & $1992-2000$ \\
\hline & & Holcim & $1992-2001$ \\
\hline & & Motor-Columbus & 1992 \\
\hline & & Sopraceneria & $1992-1999$ \\
\hline & & Zueblin & $1995 / 1996$ \\
\hline \multirow[t]{5}{*}{ Rauh } & Markus & Alusuisse & $1995-1999$ \\
\hline & & Interroll & $1998-2001$ \\
\hline & & Leica & 2001 \\
\hline & & Swisscom & $2000 / 2001$ \\
\hline & & Unaxis & 2001 \\
\hline \multirow[t]{7}{*}{ Ruepp } & Rene & Ascom & $1992-1999$ \\
\hline & & ATEL & 1996-1999 \\
\hline & & Forbo & $1992-1999$ \\
\hline & & Motor-Columbus & 1992-1999 \\
\hline & & Rieter & 1996-1999 \\
\hline & & SBG & 1994-1997 \\
\hline & & Unaxis & $1992-1996$ \\
\hline \multirow[t]{3}{*}{ Sarasin } & Beat & Bank Sarasin & $1992-2001$ \\
\hline & & Roche & 1992 \\
\hline & & SBV & 1992-1994 \\
\hline \multirow[t]{2}{*}{ Schäuble } & Rolf & Bâloise & $1995-2001$ \\
\hline & & SBV & 1995-1997 \\
\hline \multirow[t]{4}{*}{ Schmidheiny } & Jacob & Conzetta & $1992-2001$ \\
\hline & & SBV & $1992-1997$ \\
\hline & & Sulzer & $1992-2001$ \\
\hline & & Unique & $1999-2001$ \\
\hline \multirow[t]{5}{*}{ Schmidheiny } & Stephan & ABB & $1992-1997$ \\
\hline & & Landis \& Gyr & 1992-1995 \\
\hline & & Nestlé & $1992-2001$ \\
\hline & & SBG & $1992-1996$ \\
\hline & & Swatch & 1992-1994 \\
\hline \multirow[t]{7}{*}{ Schmidheiny } & Thomas & Ciment Portland & $1992-1998$ \\
\hline & & Credit Suisse Group & $1992-2001$ \\
\hline & & HCB & 1992-1994 \\
\hline & & Holcim & $1992-2001$ \\
\hline & & Sibra & 1992 \\
\hline & & Swissair & $1992-2001$ \\
\hline & & Think Tools & 2001 \\
\hline
\end{tabular}


Xstrata

1992-1994, 1998-2001

\begin{tabular}{|c|c|c|c|}
\hline Schneider- & Johann & Mikron & $1992-2001$ \\
\hline \multirow[t]{2}{*}{ Ammann } & & SBG & 1994-1997 \\
\hline & & Swatch & $2000 / 2001$ \\
\hline \multirow[t]{3}{*}{ Schweizer } & Rolf Walter & Clariant & $1996-2001$ \\
\hline & & Sandoz & 1992-1995 \\
\hline & & SBV & 1994-1997 \\
\hline \multirow[t]{6}{*}{ Senn } & Nikolaus & Alusuisse & 1992 \\
\hline & & Intershop & 1992 \\
\hline & & Landis \& Gyr & 1992 \\
\hline & & Richemont & $1992-2001$ \\
\hline & & SBG & $1992-1996$ \\
\hline & & Winterthur & $1992-1996$ \\
\hline \multirow[t]{2}{*}{ Sigg } & Hans-Peter & Bâloise & $1992-2000$ \\
\hline & & SBG & 1992 \\
\hline \multirow[t]{6}{*}{ Spälti } & Peter & Centerpulse & $1998-2001$ \\
\hline & & Credit Suisse Group & $1999 / 2000$ \\
\hline & & Rieter & 1993-1999 \\
\hline & & SBG & $1992-1996$ \\
\hline & & Sulzer & $1992-2001$ \\
\hline & & Winterthur & $1992-1996$ \\
\hline \multirow[t]{4}{*}{ Spoerry } & Vreni & Credit Suisse Group & $1992-2001$ \\
\hline & & Nestlé & $1994-2001$ \\
\hline & & Swissair & $1992-2001$ \\
\hline & & Zürich Versicherungen & $1992-1996$ \\
\hline \multirow[t]{4}{*}{ Stähelin } & Gaudenz & Bâloise & $1994-2001$ \\
\hline & & Barry Callebaut & $1999-2001$ \\
\hline & & Jelmoli & $1992-1997$ \\
\hline & & Swissair & $1992-2001$ \\
\hline \multirow[t]{4}{*}{ Studer } & Robert & Nestlé & $1994-1998$ \\
\hline & & SBG & 1997 \\
\hline & & Schindler & $1992-2001$ \\
\hline & & Swissair & $1992-1998$ \\
\hline \multirow[t]{3}{*}{ Tanner } & Ernst & Adecco & 2001 \\
\hline & & Lindt \& Sprüngli & $1995-2001$ \\
\hline & & Swatch & 1996-2001 \\
\hline \multirow[t]{4}{*}{ Tschopp } & Theodor & Alusuisse & $1996-2000$ \\
\hline & & Ciba SC & $1998-2000$ \\
\hline & & Credit Suisse Group & $1994-2000$ \\
\hline & & PSP Swiss Property & 2001 \\
\hline \multirow[t]{3}{*}{ van Wachem } & Lodewjik & $A B B$ & $1997-2000$ \\
\hline & & Credit Suisse Group & 1994-1996 \\
\hline & & Zürich Versicherungen & $1995-2001$ \\
\hline \multirow[t]{2}{*}{ Vasella } & Daniel & Credit Suisse Group & $1998-2001$ \\
\hline & & Novartis & $1997-2001$ \\
\hline \multirow[t]{6}{*}{ von der Crone } & Hugo & Bank Leu & $1992 / 1993$ \\
\hline & & Credit Suisse Group & $1992-1994$ \\
\hline & & EG Laufenburg & 1992-1999 \\
\hline & & Elektrowatt & $1992-1997$ \\
\hline & & Generali & $1992-2001$ \\
\hline & & KW Laufenburg & $1992-1998$ \\
\hline
\end{tabular}




\begin{tabular}{|c|c|c|c|}
\hline \multirow[t]{3}{*}{ von Finck } & \multirow[t]{3}{*}{ August } & Alusuisse & $1994-2000$ \\
\hline & & Mövenpick & $1994-2001$ \\
\hline & & SGS & $2000 / 2001$ \\
\hline \multirow[t]{6}{*}{ Zbinden } & \multirow[t]{6}{*}{ Paul } & Credit Suisse Group & $1993 / 1994$ \\
\hline & & Elektrowatt & 1994-1997 \\
\hline & & Escor & $1996-2001$ \\
\hline & & Feldschlösschen & 1994-1996 \\
\hline & & Sibra & 1994-1996 \\
\hline & & Sika & 1992-1994 \\
\hline \multirow[t]{4}{*}{ Zumstein } & \multirow[t]{4}{*}{ Josef } & Bâloise & 1992 \\
\hline & & Credit Suisse Group & 1992 \\
\hline & & Jelmoli & 1992 \\
\hline & & Sandoz & 1992-1994 \\
\hline
\end{tabular}

